\title{
Meta-analysis of copper and iron in Parkinson's disease brain and biofluids
}

Sian Genoud, BS, ${ }^{1}$ Alistair M. Senior, PhD, ${ }^{2,3}$ Dominic J. Hare, $\mathrm{PhD}^{4,5,6}$ and Kay L. Double, $\mathrm{PhD}^{1}$

${ }^{1}$ Brain and Mind Centre and Discipline of Pharmacology, Faculty of Medicine and Health, The University of Sydney, Sydney, Australia.

${ }^{2}$ School of Life and Environmental Sciences and School of Mathematics and Statistics, Faculty of Science, The University of Sydney, Sydney, Australia.

${ }^{3}$ Charles Perkins Centre, The University of Sydney, Sydney, Australia.

${ }^{4}$ Melbourne Dementia Research Centre at The Florey Institute of Neuroscience and Mental Health and The University of Melbourne, Melbourne, Australia.

${ }^{5}$ Department of Medicine, Royal Melbourne Hospital at The Peter Doherty Institute for Infection and Immunity, The University of Melbourne, Melbourne, Australia.

${ }^{6}$ Department of Clinical Pathology, Melbourne Medical School, The University of Melbourne, Australia.

Corresponding author for submission: A/Prof Kay L. Double, Brain and Mind Centre, 94100 Mallett Street, Camperdown, NSW 2050, Australia, e-mail: kay.double@sydney.edu.au

Word Count: 3883

This is the author manuscript accepted for publication and has undergone full peer review but has not been through the copyediting, typesetting, pagination and proofreading process, which may lead to differences between this version and the Version of Record. Please cite this article as doi: $10.1002 / \mathrm{mds} .27947$

This article is protected by copyright. All rights reserved. 
Running title: Copper and iron in Parkinson’s disease

Key words: Parkinson's disease, iron, copper, meta-analysis

Financial disclosure/conflict: The funding sources had no role in the design and conduct of the study; collection, management, analysis, or interpretation of the data; preparation, review, or approval of the manuscript; nor in the decision to submit the manuscript for publication. Assoc Prof Hare receives research and materials support from Agilent Technologies through the Australian Government National Health and Medical Research Council Career Development (Industry) Fellowship. No other potential conflicts are reported.

Funding sources: This study was funded by Parkinson's New South Wales, Parkinson’s Victoria, the Australian Research Council (DE180101520 to Dr Senior) and the National Health and Medical Research Council (GNT1122981 to Assoc Prof Hare). The Florey Institute of Neuroscience and Mental Health acknowledge the strong support from the Victorian Government and in particular the funding from the Operational Infrastructure Support Grant.

This article is protected by copyright. All rights reserved. 


\section{ABSTRACT}

Background: Variations in study quality and design complicate interpretation of the clinical significance of consistently reported changes in copper and iron levels in human Parkinson's disease brain and biofluids.

Methods and findings: We systematically searched literature databases for quantitative reports of biometal levels in the degenerating substantia nigra (SN), CSF, serum and plasma in Parkinson's disease compared with healthy age-matched controls and assessed the quality of these publications. The primary outcomes of our analysis confirmed SN copper and iron levels are decreased and increased, respectively, in the Parkinson's disease brain. We applied a novel Quality Assessment Scale for Human Tissue to categorize the quality of individual studies and investigated the effects of study quality on our outcomes. We undertook a random-effects meta-analysis and meta-regression subgroup analysis. In the 18 eligible studies identified (211 Parkinson’s disease, 215 control) SN copper levels were significantly lower ( $d,-2.00$; 95\% CI, -2.81 to -1.19; $P<0.001)$ and iron levels significantly higher $(d$, 1.31; CI $0.38-2.24 ; P<0.01)$ in Parkinson's disease. No changes were detected in CSF, serum or plasma for any metals (29 studies; 2443 Parkinson's disease and 2183 control cases) except serum iron which was lower in Parkinson’s disease (14 studies; 1177 Parkinson’s disease and 1447 controls).

Conclusion: Reductions in copper levels and elevations in iron are confirmed as characteristic of the degenerating SN of Parkinson's disease. Iron in serum is also changed, but in the opposite direction to that in the $\mathrm{SN}$ and to a lesser extent. 


\section{INTRODUCTION}

Motor dysfunction in Parkinson's disease (PD) is caused by the progressive loss of dopaminergic neurons, particularly in the ventral substantia nigra (SN), and the consequent diminution of dopaminergic transmission by the nigrostriatal pathway. As available antiparkinsonian therapies do not modify this disease process, ${ }^{1}$ identifying pathophysiological processes that may be effective targets for neuroprotective therapies is a major research focus. Over the past decade, dysfunction of metal-catalyzed pathways in neurodegenerative disorders has attracted substantial interest ${ }^{2}$. Genetic disorders involving dysregulation of pathways that require copper and iron, such as Wilson's disease, ${ }^{3}$ Menkes disease, ${ }^{4}$ and neurodegeneration with brain iron accumulation ${ }^{5}$ illustrate the essential roles played by copper and iron in neuronal health and the pathologic consequences of major shifts in their levels.

Increased local iron levels in the brains of people with PD were first noted during the 1920 s, ${ }^{6}$ and abnormal iron accumulation in sites of neurodegeneration has since been regularly reported. More recently, reduced copper concentrations, together with increased iron levels, have been reported in degenerating brain regions in PD. ${ }^{7}$ However, the quality of such studies has been heterogeneous and affected by problems including small and diverse sample cohorts, the possibility of post mortem artefacts caused by metal chelation or contamination, and differences in preparation procedures and analytic techniques. Altered metal levels in 
biofluids, such as CSF, sera and plasma, have also been described, ${ }^{8}$ but the relationship between these changes and those of brain metals is unclear.

Pharmacological modulation of brain metal levels in PD is being investigated in several clinical trials, including the administration of the orally bioavailable, blood brain barrierpermeable copper complex $\mathrm{Cu}(\mathrm{II}) \mathrm{ATSM}$ for restoring normal copper levels in the $\mathrm{SN}^{9}$ (phase 1; ClinicalTrials.gov Identifier: NCT03204929), and of the chelator deferiprone to reduce iron levels ${ }^{10}$ (phase 2; ClinicalTrials.gov Identifier: NCT02728843). Confirming copper and iron changes in regions that degenerate in PD would support these therapeutic strategies.

To overcome limitations of individual observation studies and to increase the statistical power of analysis, we undertook a systematic review and meta-analysis of all English language publications on SN and biofluid copper and iron levels in PD. Meta-analysis is useful for estimating the degree to which variation in the outcomes of different studies are related to limited sample sizes (sampling variance), and methodology (true heterogeneity). Further, we evaluated study bias and the validity of methodological design and reporting in individual post mortem and biofluid studies with a scale we specifically developed for assessing the quality of biochemical studies of human samples. Study quality provides clinometric information that enables statistical adjustment of individual datasets in subsequent subgroup meta-analyses.

\section{METHODS AND MATERIALS}

This article is protected by copyright. All rights reserved. 


\section{Search Strategy and Study Selection}

Author SG searched Web of Science, EMBASE and MEDLINE for the terms “copper”/“Cu” or “iron”/"Fe” or “metal*” AND “Parkinson*” on 13 September 2017. All articles in English published before the search date were initially included in the analysis. No search restrictions regarding analytic methods or experimental approach (i.e., in vitro or in vivo) were applied. We undertook this systematic review and meta-analysis according to the guidelines of the Preferred Reporting Items for Systematic Reviews and Metal-analyses (PRISMA) statement (Fig. 1). ${ }^{11}$

\section{Data extraction}

The initial search identified 14,487 articles. After duplicates were excluded, 9,026 unique articles were pre-screened by two authors (SG, KLD) according to pre-defined exclusion criteria (quantitative measurements of copper, iron, zinc or manganese in human post-mortem SN tissue, CSF, sera or blood in PD patients); 8929 records were excluded (Figure 1), and 97 articles further assessed for eligibility:

- Forty-one articles reporting metal concentrations in post mortem SN tissue: 23 articles were excluded as ineligible according to pre-defined criteria (Supplementary Table 2); 18 articles describing data for 211 PD and 215 control brains were included in our meta-analysis of post mortem SN reports (Supplementary Table 3).

This article is protected by copyright. All rights reserved. 
- Fifty-six articles reporting metal concentrations in CSF, serum, or plasma samples: 27 were excluded as ineligible according to pre-defined criteria (Supplementary Table 4); 29 articles describing data from 2443 PD and 2183 control cases were included in our meta-analysis of biofluid metal levels (Supplementary Tables 5 to 7).

The characteristics of each study (numbers of samples; the mean metal levels, with standard deviations) were extracted for analysis. The primary outcome was difference in metal concentrations (iron and copper) between PD and control tissues. Differences in zinc and manganese levels were also examined as secondary outcomes, as associations between these biometals and PD pathology have been discussed. ${ }^{12,13}$

The included articles described samples from tissue donors from a range of ethnic backgrounds and continents (Europe, Asia, Australia, the Americas). No donors contributed tissue to both the brain and biofluid metal datasets. Studies reporting metal concentrations in SN tissue often also reported data for other brain regions (frontal, occipital, temporal cortices; cerebellum; striatum). However, given the small sample sizes for these other regions and the significance of the SN for PD etiology, we restricted our analysis of brain tissue levels to the SN.

\section{The Quality Assessment Scale for Biochemical Analysis of Human Samples}

There is no gold standard technique for assessing metal levels in human tissues. Despite our rigorous exclusion criteria, the included studies differed in design; differences in techniques 
for measuring minor and trace metals may have affected data quality. ${ }^{14}$ The sensitivity of the various analytic options differs markedly; for example, the sensitivity of Mössbauer spectroscopy is relatively low, ${ }^{15}$ while synchrotron-microprobe $\mathrm{x}$-ray fluorescence is the most sensitive technique, although it is usually applied to single cell, rather than regional, analyses. As biometals are extremely sensitive to local environmental changes, ${ }^{16}$ we also included studies that assessed both fresh frozen and formalin-fixed tissues to compare the effect of tissue preparation.

Protocols for assessing the quality of quantitative data from human tissue analyses have not been published. We therefore developed the Quality Assessment Scale for Biochemical Analysis of Human Samples (see Supplementary Materials for detail and $R$ code), based on the Cochrane Collaboration-approved Newcastle-Ottawa scale for assessing the quality of nonrandomised studies in meta-analyses. ${ }^{17}$ Our scale scores aspects of study design, sample selection, tissue quality, detection method, and statistical analysis. A maximum 15 points can be allotted: a study with 11-15 points is deemed high quality, 6-10 points moderate quality, and 0-5 points low quality (Table 1). We applied our scale to both SN tissue and biofluid studies. Test-retest reliability was assessed by one author (SG) who applied the scale to both the SN and biofluids datasets at two separate time-points on one-third of studies. Inter-rater reliability was assessed by two authors (SG, KLD) by independently applying the scale to the same studies.

\section{Data analysis}


All analyses were performed with the metafor package for R 3.4 (R Core Team). The differences between the means of the two groups in each study (in units of pooled SD), expressed as effect size, were assessed with Hedges' $d$ statistic, ${ }^{18}$ calculated with the 'escalc' function in metafor. ${ }^{19}$ Effect sizes were calculated so that a negative value indicated that metal concentration was lower in the PD than the control tissue group. Standard interpretive benchmarks for Hedges’ $d$ were applied $(0.2=$ small, $0.5=$ medium, $0.8=$ large $)$.

Data for metal concentrations in post mortem SN tissue, CSF, serum, and plasma were analyzed separately. For each dataset, a random-effects meta-analysis (REMA) model was applied using the 'rma' function in metafor to estimate the overall magnitude and statistical significance of an effect. The REMA model was preferred to a fixed effects model because the included studies included potential sources of heterogeneity arising from differences in donor cohorts and analytic methods. A REMA model weights individual studies according to both the inverse of within-study variance (square root of the SE for sampling variances), and between-study variance, which also depends on sample size. Variation in effect sizes that could not be attributed to sampling variance, or 'heterogeneity', was also estimated by each REMA model. Heterogeneity statistics quantify the degree to which the results of different studies differ on factors other than sampling (e.g., analytic methods). Each REMA estimates whether effect size heterogeneity was statistically significant with the $Q$ statistic. We also report the proportion of variance in reported effects not attributable to sampling variance 
alone (and therefore ascribed to differences in analytic approach) as $I^{2,20,21}$ values below 25\% were deemed low, 25-75\% medium, and more than 75\% high heterogeneity.

Random effects meta-regression (REMR) and subgroup analyses were conducted on datasets categorized by study quality according to our scale (Supplementary Table 8), patient age, sex, and post-mortem interval (SN tissue only to assess observed data heterogeneity. Subgroup analyses also assessed overall effect sizes as a function of analytic technique, tissue preparation method, and the significance of differences between subgroups in REMA.

\section{Assessment of Publication Bias}

Publication bias was investigated when ten or more datasets reported data on the same variable $^{22}$ by Egger's regression ('regtest' function in metafor ${ }^{23}$ and visualized as funnel plots (Supplementary Fig. 1-3). Where Egger’s regression detected statistically significant evidence of bias $(P<0.05)$, a trim and fill analysis was applied (metafor 'trimfill' function) to estimate the number, magnitude, and precision of potentially missing effect sizes, and to calculate a revised overall effect. ${ }^{21,24}$ The statistical methodology is described in greater detail in the Supplementary Materials.

\section{RESULTS}

Demographic characteristics of tissue donors: age

This article is protected by copyright. All rights reserved. 
The mean age of SN tissue donors was 76.1 years (SD, 3.7 years) for donors with PD, 74.8 years (SD, 5.7 years) for control donors; the difference in mean age between the two groups was 1.6 years (SD, 5.0 years). The mean age of CSF donors was 65.0 years (SD, 3.5 years) for donors with PD, 61.0 years (SD, 5.3 years) for control donors; the difference in mean age was 5.9 years (SD, 11.9 years). The mean age of serum donors was 64.3 years (SD, 4.4 years) for donors with PD, 60.0 years (SD, 5.3 years) for control donors; the difference in mean age was 3.7 years (SD, 4.0 years). The mean age of plasma donors was 61.5 years (SD, 5.6 years) for donors with PD, 58.4 years (SD, 13.9 years) for control donors; the difference in mean age between the two groups was 3.1 years (SD, 0.8 years). Further demographic data are reported in Supplementary Tables 4-7.

\section{Quality Assessment Scale for Biochemical Analysis of Human Samples}

Test-retest reliability of the scale was high (correlation, 0.99; $P<0.0001$ ) with $91 \%$ agreement $(n, 11 ; \kappa, 0.889 ;-P<0.0001)$. Inter-rater reliability for two independent raters was also high (correlation, $0.99 ; P<0.0001)$, with $82 \%$ agreement $(n, 11 ; \kappa, 0.778 ; P<0.0001)$. Seven of 18 human post mortem tissue studies were deemed high quality, nine moderate quality and two limited quality studies. Five of the 12 CSF studies were categorized as high quality studies, seven as moderate quality. Seven of 17 serum studies were deemed high quality, seven moderate quality and one low quality studies. It should be acknowledged that one factor the Quality Assessment Scale cannot account for is the analytical validity of the method used. Blank concentrations, limits of analysis, and potential contamination sources 
are not typically reported. This may have the effect of masking changes in studies that did not report changes due to the sensitivity of the method used.

\section{Copper levels in the substantia nigra are reduced in PD}

Nine of 18 reports on post mortem SN tissue included quantitative data on copper levels (PD, 127; control, 134 samples); six reported that copper levels were significantly lower in PD than control samples, three studies found no significant difference (Figure 2A). Overall, copper levels were significantly lower in PD samples ( $d$, -2.00 [large effect]; 95\% CI, -2.81 to $-1.19 ; P=0.0051$; Fig. 2A), but study heterogeneity was substantial $\left(I^{2}, 83.9 \% ; Q, 59.9 ; P\right.$ $<0.0001)$. The effect size was similar in the six high quality studies that measured copper $(d$, $-2.05 ; 95 \%$ CI, -2.95 to $-1.16 ; P<0.0001)$ and the three moderate quality studies $(d,-1.84$; $95 \% \mathrm{CI},-4.04$ to $0.36 ; P=0.10)$. The four studies that employed inductively coupled plasma-mass spectrometry (ICP-MS) $(d,-2.42$; 95\% CI, -3.92 to $-0.92 ; P=0.0016)$ and the three atomic absorption spectroscopy studies (AAS; $d,-2.25 ; 95 \%$ CI, -4.02 to $-0.47 ; P=$ 0.013 reported the largest effect sizes; the effect sizes of the three microprobe X-ray fluorescence/emission and one Mössbauer spectroscopy study were not statistically significant (Supplementary Table 10. The effect sizes in the eight studies using fresh tissue were significant $(d,-2.06$; $95 \% \mathrm{CI},-3.01$ to $-1.12 ; P<0.0001)$, in contrast to the two studies using formalin-fixed tissue $(d,-1.76 ; 95 \% \mathrm{CI},-3.72$ to $0.21 ; P=0.07)$. Effect sizes were not influenced by age, sex, or post mortem interval (Supplementary Table 10), consistent with 
within-study matching for these variables. No publication bias was identified (Supplementary Figure 1A).

\section{Iron levels are elevated in the in the substantia nigra in PD}

All but one of the 18 post mortem SN tissue articles reported quantitative iron levels (PD, 203; control, 221 samples); 13 studies reported that iron levels were significantly higher in PD than control samples, four studies found no significant difference (Figure 2B). Overall, iron levels were significantly higher in PD samples $(d, 1.22 ; 95 \% \mathrm{CI}, 0.36-2.09 ; P=0.0055$, but study heterogeneity was substantial ( $I^{2}, 92.8 \%$; $Q, 148 ; P<0.0001 ;$ Fig. 2B). The effect size was slightly larger if two low quality studies (PD, 22; control, 30 samples) were excluded ( $d, 1.35$; 95\% CI, 0.37-2.33; $P<0.007)$, and was also greater for studies using unfixed, fresh tissue (14 studies: $d, 1.48$; 95\% CI, 0.50-2.46; $P<0.003$ ). Within analytical technique subgroups, no technique demonstrated a significant difference in effect size (Supplementary Table 10). The between-group difference in post mortem interval significantly influenced effect size, with increasing difference in interval associated with larger effects (slope for $d, 0.09$; 95\% CI, 0.04-0.15; $P<0.0008$ ), while disease duration, donor age, and sex did not influence effect size (Supplementary Table 10). Evidence of publication bias was statistically significant $(P<0.0001$; Supplementary Fig $1 \mathrm{~B})$, but trim and fill analyses did not alter the overall effect.

\section{Zinc levels in the substantia nigra are unchanged in PD}

This article is protected by copyright. All rights reserved. 
Three of seven studies that assessed zinc levels in the SN reported that levels were significantly higher in PD than control samples (Figure 2C). Meta-analysis of all seven studies indicated that zinc levels were similar in PD and healthy aged control samples $(d$, 0.64, $95 \% \mathrm{CI}-0.28$ to $1.56, P=0.18$; Fig. $2 \mathrm{C}$ ). Analysis of the four high quality studies found no difference (Fig. 2C), but the effect size for the three moderate quality studies was significant $(d, 1.83,95 \% \mathrm{CI}, 0.34-3.32, P=0.016)$. Age, post mortem interval, sex, and disease duration did not significantly influence the effect (Supplementary Table 9). Publication bias was statistically significant $(P=0.0072)$; Supplementary Fig $1 \mathrm{C})$, but trim and fill analyses estimated no missing studies.

\section{Biometal levels in CSF, serum, and plasma in people with PD}

Eight of the ten studies that assessed copper levels in CSF found no difference in levels between samples from donors with or without PD, while one study each found them to be significantly lower or higher in PD donors (Figure 3A). Assessments of CSF iron levels in CSF were also mixed, with four reporting they were lower in PD, two that they were higher, and three that they were similar to those of control donors. Of seven studies of CSF zinc levels three found that they were lower in PD, one that they were higher, and three that they were similar to those of control donors (Figure 3C). Of seven studies assessing CSF manganese levels, one reported that they were higher in PD, six that levels were similar in PD and control samples (Figure 3D). Overall (11 CSF studies; PD, 576; control, 293 
samples) there copper, iron, zinc, and manganese levels in PD were not significantly different to those of control donors (Fig. 3). The only statistically significant differences in effect size by study quality were that iron $\left(d,-0.53\right.$; $95 \% \mathrm{CI},-0.79$ to $\left.-0.26 ; P<0.0001 ; I^{2}=23.0 \%\right)$ and zinc levels in four high quality studies $\left(d,-0.52\right.$; $95 \%$ CI, -0.82 to $-0.23 ; P=0.0004 ; I^{2}=0$ ) were lower in PD than control samples. There was no evidence of publication bias (Supplementary Figure 3).

Of the 15 studies that assessed serum copper, nine found that levels were lower in PD, one that they were higher, and five that they were similar in PD and control samples. Overall, serum copper levels were similar in PD and control samples (14 studies: PD, 1235; control, 1132 samples); study heterogeneity was high $\left(I^{2}, 98.8 \% ; Q, 603 ; P<0.0001\right)$ but Figure 4A).

Five serum iron studies reported that they were lower in PD, one that they were higher, and eight that they were similar in PD and control samples (Figure 4B). Overall, serum iron levels were significantly lower in PD samples (14 studies [six high, seven moderate, one low quality]: PD, 1177; control 1447 samples; $d,-0.77$; CI, -1.43 to $-0.10 ; P=0.024$ Fig. 4B); excluding the low quality study (35 PD, 33 control samples) rendered the effect nonsignificant ( $d,-0.63$; CI, -1.28 to $0.03 ; P=0.06$; Fig $4 C$ ), as did including only the six high quality studies (PD, 701; control, 761 samples: $d$, -0.97 ; CI, -2.31 to $0.57 ; P=0.24$; Fig. 4C). Meta-regression analyses indicated that serum iron level increased with age (slope, 0.18 , 95\% CI, 0.06-0.22; $P<0.005)$; but the ages of donors in the PD and control cohorts did not 
differ, nor between these cohorts when high, moderate and low quality studies were individually tested.

Four of 12 studies that assessed serum zinc found that levels were lower in PD, and eight that they were similar in PD and control samples (Figure 4D). Of the seven studies that assessed serum manganese, one found that levels were lower in PD and one that they were reduced (Figure 4E). Overall, serum zinc and manganese levels were similar in PD and control samples (Fig. 4D,E). There was no evidence of publication bias for serum metal studies (Supplementary Figure 3).

Meta-analysis of plasma metal data was limited by the low number of studies that met our selection criteria (five) and data heterogeneity for all three metals assessed (copper: $d, 0.0$, 95\% CI -1.82 to $1.81, P=0.996, I^{2}=98.6 \%$; iron: $d, 0.35,95 \% \mathrm{CI},-1.07$ to $1.77, P=0.63$, $I^{2}=96.4 \%$; zinc, $d,-0.40,95 \% \mathrm{CI}-0.78$ to $\left.-0.01, P=0.043, I^{2}=0 \%\right)$. One copper and one zinc study were deemed low quality, but their omission did not change our findings.

(Supplementary Table 9). There was no evidence of publication bias for plasma studies.

\section{No association between copper and iron levels in the substantia nigra and in biofluids}

Meta-regression indicated that the lower SN copper levels in PD samples were not correlated with lower biofluid copper levels in age-matched individuals. Similarly, higher SN iron 
levels in PD samples were not mirrored by higher biofluid iron levels, before or after adjusting for age (Supplementary Figure 4).

\title{
DISCUSSION
}

In the first meta-analysis of SN copper and zinc levels in people with $\mathrm{PD}$, and in accordance with that a previous meta-analysis of iron $^{32}$, we found evidence that copper levels are consistently lower than in healthy controls, and confirmed that iron levels are significantly higher. The magnitudes of these differences were similar, but in opposite directions. The studies we analyzed included donors of different ethnic backgrounds from multiple continents, suggesting our findings can be generalized to people with PD worldwide.

\begin{abstract}
Although studies of copper and iron levels in PD consistently report changes restricted to degenerating brain regions, particularly the $\mathrm{SN},{ }^{25,26}$ the available data did not permit meaningful analysis of metal level changes in other brain regions. The measured differences in SN iron and copper levels in PD may reflect redistribution of metals within the SN or between the SN and other tissues. ${ }^{27}$ Intranigral redistribution would be consistent with the significantly higher levels of intraneuronal iron and lower levels of intraneuronal copper in in PD we have described. ${ }^{7}$
\end{abstract}

Despite the high degree of study heterogeneity, the magnitudes of the overall changes our meta-analysis identified are consistent with those we and others have found in studies of 
within-dopaminergic neuron metal concentrations in PD. ${ }^{26,28}$ We have found these changes in cases of probable pre-clinical disease, which suggests that iron and copper levels change at the cellular level early in the disease process. ${ }^{29}$ These findings, however, do not provide unequivocal evidence for their etiological significance. Nevertheless, like the discovery of markedly reduced dopamine levels that motivated the first trials of dopamine replacement therapy in the early 1960s, our findings provide strong empirical evidence of specific changes in the primary region of neurodegeneration in $\mathrm{PD}$, supporting the rationale of current clinical trials investigating these changes as targets for disease modification in PD. ${ }^{9,10}$

Our data support the hypothesis that increased SN iron levels are at least a biomarker of the disease process, including in pre-clinical PD. Elevated SN iron levels detected by functional ultrasound imaging detection have been proposed a risk marker for pre-clinical PD by the International Parkinson and Movement Disorders Society Task Force, ${ }^{30}$ and quantifying SN iron by MRI imaging is being investigated both as a biomarker of PD and of disease progress. ${ }^{31}$ We did not include functional imaging of brain iron in PD in our analysis, as this was recently the subject of meta-analysis. ${ }^{32}$ Copper and zinc cannot currently be assessed in vivo with functional imaging.

We also examined whether the effect sizes for changes in copper and iron levels in the post mortem SN were matched by changes in intra vitam CSF, serum, and plasma levels in people with PD. Given the close physical contacts between the brain, CSF, and capillary network, changes in biometal levels in the more accessible biofluids could potentially be useful 
biomarkers of central disease processes. ${ }^{33}$ Our analysis indicated that biofluid copper levels are within normal limits in PD, but serum iron levels are lower than in age-matched healthy control people. One major limitation of our analysis, however, was that brain and biofluid tissue samples were not sourced from the same donors; a study that compared both sample types within individuals would be invaluable, but investigations of this type have not been reported. Biofluid metal levels are derived from various sources however, and therefore circulating metal levels may therefore not be useful for estimating brain metal levels.

We initially weighted individual studies according to donor group size and variance, but other design aspects, such as analytic technique and tissue preparation, significantly influenced effect size, suggesting such factors should be carefully considered during experimental design. In contrast, other variables reported to influence biometal levels, including sex ${ }^{34}$ and post-mortem interval ${ }^{35}$ did not have significant effects. No robust evidence that medications influence biometal levels in the human brain or biofluids has been reported. Monoamine oxidase inhibitors prescribed for patients with PD may have ironchelating properties ${ }^{36}$ that may be relevant to reports of reduced serum iron levels in PD. ${ }^{37}$ Little is known, however, about the effects on brain biometal levels of other dopamine replacement therapies or of other medications frequently taken by older people.

\section{Conclusion}

This article is protected by copyright. All rights reserved. 
Copper levels are significantly lower and iron levels significantly higher in post mortem SN tissue from PD patients than from healthy controls. Serum iron levels, however, are lower in PD than in samples from healthy age-matched controls. Our findings provide evidence for marked changes in iron and copper levels in the major site of neurodegeneration in PD. These findings warrant further investigation to define the significance of SN biometal levels for the pathophysiology of PD, and to determine whether therapies targeting these metals would be both feasible and clinically useful.

\section{Author contributions}

S.G., D.J.H. and K.L.D. designed the study. S.G. performed the literature search, did the statistical analysis with the help of A.M.S., and wrote the first version of the report with the help of D.J.H. and K.L.D.

\section{Financial Disclosures of all authors (for the preceding 12 months)}

S.G. is a PhD candidate at the University of Sydney and recipient of a Research Training Program stipend. A.M.S. is an employee of the University of Sydney and is funded by an Australian Research Council Discovery Early Career Researcher Award. D.J.H. is an employee of the Florey Institute of Neuroscience and Mental Health and is supported by a National Health and Medical Research Council Career Development Fellowship. K.L.D. is an employee of the University of Sydney and supported by intramural funds. The authors have 
no other financial interests to declare.

This article is protected by copyright. All rights reserved. 


\section{References}

1. Kalia LV, Lang AE. Parkinson’s disease Lancet Neurology 2015;386:896-912.

2. Barnham KJ, Bush AI. Biological metals and metal-targeting compounds in major neurodegenerative diseases. Chemical Society reviews 2014;43(19):6727-6749.

3. Bandmann O, Weiss KH, Kaler SG. Wilson's disease and other neurological copper disorders. Lancet Neurol 2015;14(1):103-113.

4. Kaler SG. ATP7A-related copper transport diseases-emerging concepts and future trends. Nature Reviews Neurology 2011;7(1):15-29.

5. Fleming RE, Ponka P. Iron Overload in Human Disease. New England Journal of Medicine 2012;366(4):348-359.

6. Spatz H. Über den Eisennachweis im Gehirn, besonders in Zentren des extrapyramidal-motorischen systems (On the visualization of iron in the brain, especially in the centers of the extrapyramidal motor system). Z Ges Neurol Psychiat 1922;77:261-390.

7. Davies KM, Bohic S, Carmona A, et al. Copper pathology in vulnerable brain regions in Parkinson's disease. Neurobiology of Aging 2014;35(4):858-866.

8. Bocca B, Alimonti A, Senofonte O, et al. Metal changes in CSF and peripheral compartments of parkinsonian patients. Journal of the neurological sciences 2006;248(12):23-30.

9. Hung LW, Villemagne VL, Cheng L, et al. The hypoxia imaging agent CuII(atsm) is neuroprotective and improves motor and cognitive functions in multiple animal models of Parkinson’s disease. Journal of Experimental Medicine 2012;209(4):837-854.

10. Moreau C, Duce JA, Rascol O, et al. Iron as a therapeutic target for Parkinson's disease. Movement Disorders 2018;33(4):568-574.

11. Moher D, Liberati A, Tetzlaff J, Altman DG, The PRISMA Group. Preferred reporting items for systematic reviews and meta-analyses: the PRISMA statement. PLoS medicine 2009;6(7):e1000097.

12. Du K, Liu MY, Zhong X, Wei MJ. Decreased circulating Zinc levels in Parkinson's disease: a meta-analysis study. Sci Rep 2017;7(1):3902.

13. Olanow CW. Manganese-induced parkinsonism and Parkinson's disease. Ann N Y Acad Sci 2004;1012:209-223.

14. Jensen A, Kjelgaard-Hansen M. M ethod comparison in the clinical laboratory. Veterinary Clinical Pathology 2006;35(3):276-286. 
15. Hare DJ, Gerlach M, Riederer P. Considerations for measuring iron in post-mortem tissue of Parkinson's disease patients. J Neural Transm 2012;119(12):1515-1521.

16. Hare DJ, George JL, Bray L, et al. The effect of paraformaldehyde fixation and sucrose cryoprotection on metal concentration in murine neurological tissue. Journal of Analytical Atomic Spectrometry 2014;29(3):565-570.

17. Wells GA, Shear B, O'Connell D, et al. The Newcastle-Ottawa Scale (NOS) for assessing the quality of nonrandomised studies in meta-analyses: The Ottawa Hospital Research Institute, 2004.

18. Hedges LV, methods JL. Fixed-and random-effects models in meta-analysis. Psychological Methods 1998;3(4):486-504.

19. Viechtbauer W. Conducting meta-analysis in R with the metafor package. Journal of Statistical Software 2010;36(3):1-48.

20. Senior AM, Grueber CE, Kamiya T, et al. Heterogeneity in ecological and evolutionary meta-analyses: its magnitude and implications. Ecology 2016;97(12):32933299.

21. Nakagawa S, Noble DW, Senior AM, Lagisz M. Meta-evaluation of meta-analysis: ten appraisal questions for biologists. BMC Biol 2017;15(1):18.

22. Lau J, Ioannidis JP, Terrin N, Schmid CH, Olkin I. The case of the misleading funnel plot. BMJ 2006;333(7568):597-600.

23. Egger M, Davey Smith G, Schneider M, Minder C. Bias in meta-analysis detected by a simple, graphical test. BMJ 1997;315(7109):629-634.

24. Duval S, Tweedie R. Trim and fill: A simple funnel-plot-based method of testing and adjusting for publication bias in meta-analysis. Biometrics 2000;56(2):455-463.

25. Genoud S, Roberts BR, Gunn AP, et al. Subcellular compartmentalisation of copper, iron, manganese, and zinc in the Parkinson's disease brain. Metallomics 2017;9(10):14471455.

26. Davies KM, Bohic S, Carmona A, et al. Copper pathology in vulnerable brain regions in Parkinson's disease. Neurobiol Aging 2014;35(4):858-866.

27. Jellinger KA. The relevance of metals in the pathophysiology of neurodegeneration, pathological considerations. Int Rev Neurobiol 2013;110:1-47.

28. Martin WR, Wieler M, Gee M. Midbrain iron content in early Parkinson disease: a potential biomarker of disease status. Neurology 2008;70(16 Pt 2):1411-1417. 
29. Berg D, Postuma RB, Adler CH, et al. MDS research criteria for prodromal Parkinson's disease. Movement Disorders 2015;30(12):1600-1611.

30. Postuma RB, Berg D. Advances in markers of prodromal Parkinson disease. Nature Reviews Neurology 2016;12(11):622-634.

31. Guan X, Xuan M, Gu Q, et al. Regionally progressive accumulation of iron in Parkinson's disease as measured by quantitative susceptibility mapping. NMR in biomedicine 2017;30(4).

32. Wang J-Y, Zhuang Q-Q, Zhu L-B, et al. Meta-analysis of brain iron levels of Parkinson's disease patients determined by postmortem and MRI measurements. Scientific reports 2016;6:36669.

33. Lewczuk P, Riederer P, O’Bryant SE, et al. Cerebrospinal fluid and blood biomarkers for neurodegenerative dementias: An update of the Consensus of the Task Force on Biological Markers in Psychiatry of the World Federation of Societies of Biological Psychiatry. World Journal of Biological Psychiatry 2017;19(4):244-328.

34. de Lau LML, Breteler MM. Epidemiology of Parkinson's disease. Lancet Neurol 2006;5(6):525-535.

35. Stan AD, Ghose S, Gao XM, et al. Human postmortem tissue: what quality markers matter? Brain Res 2006;1123(1):1-11.

36. Kupershmidt L, Weinreb O, Amit T, Mandel S, Bar-Am O, Youdim MB. Novel molecular targets of the neuroprotective/neurorescue multimodal iron chelating drug M30 in the mouse brain. Neuroscience 2011;189:345-358.

37. Pichler I, Del Greco MF, Gogele M, et al. Serum iron levels and the risk of Parkinson disease: a Mendelian randomization study. PLoS Med 2013;10(6):e1001462. 
Table 1: Scoring rubric for the Quality Assessment Scale for Biochemical Analysis of Human Samples.

\begin{tabular}{|c|c|c|}
\hline \multicolumn{3}{|c|}{ SELECTION } \\
\hline \multicolumn{3}{|c|}{ 1. Is the case definition adequate? } \\
\hline a. & Yes, both clinical and pathologically defined & 2 \\
\hline b. & Either clinical or pathologically defined & 1 \\
\hline c. & No description & 0 \\
\hline \multicolumn{3}{|c|}{ 2. Representation of population? } \\
\hline a. & Cases from multiple tissue banks or live cohorts & 2 \\
\hline b. & Tissue bank or live cohort stated & 1 \\
\hline c. & Potential for selection biases or not stated & 0 \\
\hline \multicolumn{3}{|c|}{ 3. Selection of controls } \\
\hline a. & Controls selected from same tissue banks or live population & 1 \\
\hline b. & Controls from different source or no description & 0 \\
\hline \multicolumn{3}{|c|}{ 4. Definition of controls } \\
\hline a. & No history of disease or confounding condition & 1 \\
\hline b. & Diagnosed conditions provided but effect on variable of interest unknown & 0 \\
\hline c. & No description & 0 \\
\hline \multicolumn{3}{|c|}{ COMPARABILITY } \\
\hline \multicolumn{3}{|c|}{ 1. Comparability of cases and controls on the basis of the design or analysis } \\
\hline \multicolumn{2}{|c|}{$\begin{array}{l}\text { a. Study controls for the most significant potential moderating variables } \mathbf{x} \text { and } \mathbf{y} \text { (where } \mathbf{x} \text { is the } \\
\text { most important factor) e.g. age, sex, genetic background; } 2 \text { points }\end{array}$} & 2 \\
\hline a. & Study controls for only one (most important) factor & 1 \\
\hline b. & Not controlled or not stated & 0 \\
\hline \multicolumn{3}{|c|}{ 2. Quality of tissue } \\
\hline \multicolumn{2}{|r|}{$\begin{array}{l}\text { a. Post-mortem delay is }<24 \text { hrs for post-mortem tissues; or biofluids were stored at }-20 \mathrm{C} \\
<1 \mathrm{hr} \text { after collection ( } 1 \text { point). }\end{array}$} & 1 \\
\hline b. & $>24 \mathrm{hrs}$ for post-mortem or $>1 \mathrm{hr}$ for biofluids or not defined. & 0 \\
\hline \multicolumn{3}{|c|}{ 3. Method detail } \\
\hline a. & An appropriate method applied and described & 2 \\
\hline b. & Methods and/or description limited & 1 \\
\hline c. & Inappropriate method and/or missing crucial information. & 0 \\
\hline \multicolumn{3}{|c|}{ ANALYSIS } \\
\hline \multirow{4}{*}{$\begin{array}{rr}1 . \quad A \\
\\
\quad \mathrm{a} \\
\mathrm{b} \\
\mathrm{c}\end{array}$} & iinment of exposure & \\
\hline & Blinded to case/control status & 1 \\
\hline & Unblinded. & 0 \\
\hline & No description & 0 \\
\hline \multicolumn{3}{|c|}{ 2. Appropriate and detailed statistical analysis } \\
\hline a. & Detailed and appropriate analysis & 2 \\
\hline b. & Statistical test stated & 1 \\
\hline c. & No description. & 0 \\
\hline \multicolumn{3}{|c|}{ 3. Elimination of outliers } \\
\hline a. & All participants included & 1 \\
\hline b. & Similar proportion excluded for both groups & 1 \\
\hline c. & Different exclusion or not stated & 0 \\
\hline & Maximum score: SELECTION + COMPARABILITY + ANALYSIS = & 15 \\
\hline
\end{tabular}




\section{FIGURE LEGENDS}

Figure 1: PRISMA flowchart of literature search, screening, and eligibility assessment. CSF = cerebrospinal fluid; $\mathrm{PD}=$ Parkinson’s disease; $\mathrm{SN}=$ substantia nigra .

Figure 2: Forest plots of analysis of (A) copper, (B) iron, and (C) zinc in human postmortem SN tissue. Square marker indicates effect size and statistical weight of study; horizontal lines indicate 95\% CIs. The diamond data markers represent the subtotal and overall effect size and 95\% CI. The vertical dashed line shows the summary effect estimate; the dotted line shows the line of no effect $(d=0)$. Asterisk $(*)$ represents studies identifying a reduction and $\wedge$ indicates studies reporting a significant increase. $\mathrm{df}$ : degrees of freedom. $Q$ : Cochran's $Q$.

Figure 3: Forest plots of analysis of (A) copper, (B) iron, (C) zinc, and (D) manganese in human CSF.

Figure 4: Forest plots of analysis of (A) copper, (B) iron, (C) zinc, and (D) manganese in human serum. 
\# Script to do all analyses in Genoud et al.

\# Written by Sian Genoud and Alistair Senior at the University of Sydney, 2018-2019

\# Chunks of Code are broadly split up by Figure and associated analyses, each chunk is independent of the others and can be run on its own

\# Files required in the working directory are

\# 1) Cu SN quality order.csv - Copper levels in PD and Ct substantia nigra brain tissue subgroup of study quality

\# 2) Fe SN quality order.csv - Iron levels in PD and Ct substantia nigra brain tissue - subgroup of study quality

\# 3) Zn SN quality order.csv - Zinc levels in PD and Ct substantia nigra brain tissue - subgroup of study quality

\# 4) Serum Cu quality order.csv - Copper levels in PD and Ct serum - subgroup of study quality

\# 5) Serum Fe quality order.csv - Iron levels in PD and Ct serum - subgroup of study quality

\# 6) Serum Zn quality order.csv - Zinc levels in PD and Ct serum - subgroup of study quality

\# 7) Serum Mn quality order.csv - Manganese levels in PD and Ct serum - subgroup of study quality

\# 8) CSF Cu quality data.cSv - Copper levels in PD and Ct CSF - subgroup of study quality

\# 9) CSF Fe quality order.cSv - Iron levels in PD and Ct CSF - subgroup of study quality

\# 10) CSF Zn quality order.csv - Zinc levels in PD and Ct CSF - subgroup of study quality

\# 11) Plasma Cu quality order.csv - Copper levels in PD and Ct plasma - subgroup of study quality

\# 12) Plasma Fe quality order.csv - Iron levels in PD and Ct plasma - subgroup of study quality

\# 13) Plasma Zn quality order.csv - Zinc levels in PD and Ct plasma - subgroup of study quality

\# 14) Combined Cu data 18.2.19.csv - Copper levels in PD and Ct of all tissues - subgroup of tissue type

\# 15) Fe combined data 16.2.19.csv - Iron levels in PD and Ct of all tissues - subgroup of tissue type

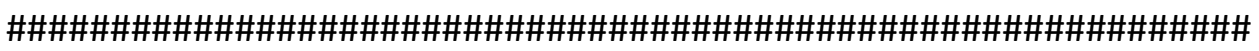

\#\#\#\#\#\#\#\#\#\# Meta-analyses For SN CU data \#\#\#\#\#\#\#\#\#\#\#\#\#\#\#\#\#

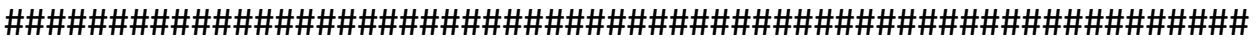

\# Deletes any potentially old info hanging around

$\mathrm{rm}($ list $=\mathrm{Is}())$

\# Set the working directory

setwd("/Users/siangenoud/Desktop/Forests")

\# Install if needs be on the first time - intall.packages("metafor") and intall.packages("plyr")

\# Load packages we need

library("metafor")

library("plyr")

This article is protected by copyright. All rights reserved. 
\# Load dataset

data<-read.csv("Cu SN quality order.csv")

\# Check the data looks good

head(data)

\# Calculate the effect size ("SMD" - Hedges d) using escalc function

\#slab function labels studies

data.ES<-escalc(measure = "SMD", n1i = PD.cases, $n 2 \mathrm{i}=$ Ct.cases, $\mathrm{m} 1 \mathrm{i}=\mathrm{PD} . \mathrm{Cu}, \mathrm{m} 2 \mathrm{i}=\mathrm{Ct}$.Cu, sd1 $\mathrm{i}=$ = PD.Cu.SD, sd2i = Ct.Cu.SD, data = data, slab=paste(Author, Year, sep=", "))

\# Fit the meta analysis for all data

MA1<-rma(yi = yi, vi = vi, data = data.ES)

summary(MA1)

\# Fit subsets for high and moderate quality studies

res.h <- rma(yi=yi, vi=vi, data=data.ES, subset=(Quality=="High"), method="REML")

res. $\mathrm{m}<-$ rma(yi=yi, vi=vi, data=data.ES, subset=(Quality=="Moderate"), method="REML")

summary(res.h)

summary(res.m)

\#Publication bias test

regtest(MA1)

\#Check for missing studies

trimfill(MA1)

\#Funnel plot

funnel(MA1)

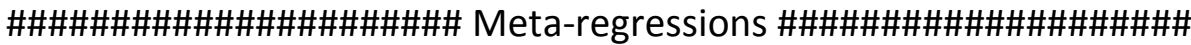

\#\#\#\#Meta-regression analysis for effect of technique

\# This first line tells you the order - by default $\mathrm{R}$ will put the first one of these categories as the intercept group

sort(unique(data.ES\$̦Technique))

\# Here you change the intercept, by changing base $=x$ at the end - here I changed it to the second in the list above

contrasts(data.ES\$Technique)<-contr.treatment(levels(data.ES\$TTechnique), base=2)

\# Overall effect of analytical technique

MRTechnique $<-r m a(y i=y i$, vi $=$ vi, data $=$ data.ES, mods $=\sim$ Technique -1$)$

summary(MRTechnique)

\# Overall effect of tissue prep

MRPrep<-rma(yi $=\mathrm{yi}, \mathrm{vi}=\mathrm{vi}$, data $=$ data.ES, $\operatorname{mods}=\sim$ Prep -1$)$

summary(MRPrep)

This article is protected by copyright. All rights reserved. 


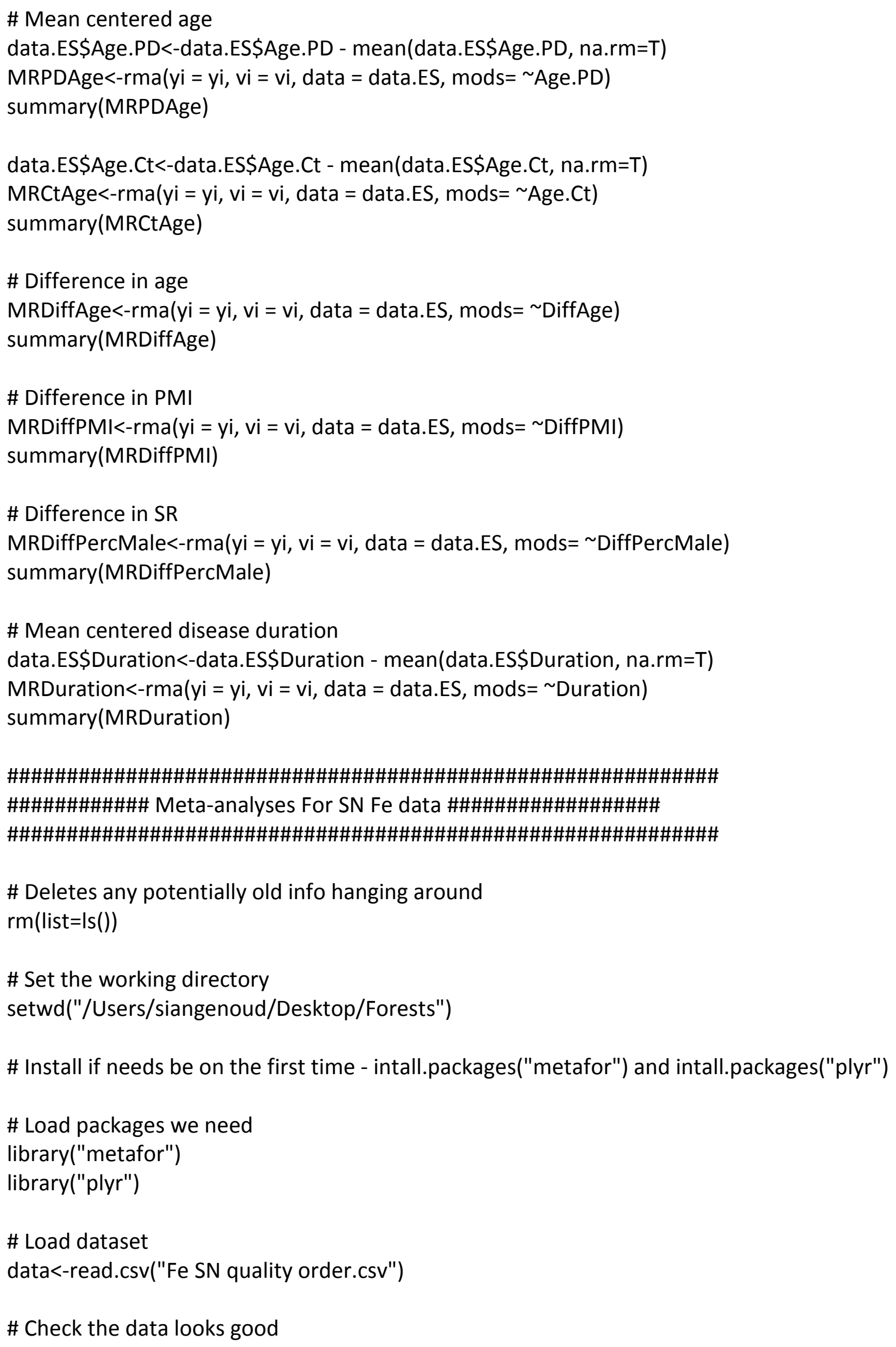


head(data)

\# Calculate the effect size ("SMD" - Hedges d) using escalc function

\#slab function labels studies

data.ES<-escalc(measure = "SMD", n1i = PD.cases, $\mathrm{n} 2 \mathrm{i}=$ Ct.cases, $\mathrm{m} 1 \mathrm{i}=\mathrm{PD} . \mathrm{Fe}, \mathrm{m} 2 \mathrm{i}=\mathrm{Ct}$.Fe, sd1 $i=$ PD.Fe.SD, sd2i = Ct.Fe.SD, data = data, slab=paste(Author, Year, sep=", "))

\# Fit the meta analysis for all data

MA1<-rma(yi = yi, vi = vi, data = data.ES)

summary(MA1)

\# Fit subsets for high and moderate quality studies

res.nL <- rma(yi=yi, vi=vi, data=data.ES, subset=(Quality=="High" | Quality == "Moderate"), method="REML")

summary(res.nL)

res.h <- rma(yi=yi, vi=vi, data=data.ES, subset=(Quality=="High"), method="REML")

summary(res.h)

res.m <-rma(yi=yi, vi=vi, data=data.ES, subset=(Quality=="Moderate"), method="REML")

summary(res.m)

res.I <- rma(yi=yi, vi=vi, data=data.ES, subset=(Quality=="Limited"), method="REML")

summary(res.l)

\#Publication bias test

regtest(MA1)

\#Check for missing studies

trimfill(MA1)

\#Funnel plot

funnel(MA1)

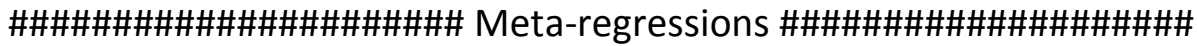

\#\#\#\#Meta-regression analysis for effect of technique

\# This first line tells you the order - by default $\mathrm{R}$ will put the first one of these categories as the intercept group

sort(unique(data.ES\$Technique))

\# Here you change the intercept, by changing base $=x$ at the end - here I changed it to the second in the list above

\# Overall effect of analytical technique

contrasts(data.ES\$Technique)<-contr.treatment(levels(data.ES\$Technique), base=2)

MRTechnique $<-$ rma $(y i=y i$, vi $=$ vi, data $=$ data.ES, mods $=\sim$ Technique -1$)$

summary(MRTechnique)

\# Overall effect of tissue prep

MRPrep<-rma(yi $=$ yi, vi $=$ vi, data $=$ data.ES, $\operatorname{mods}=\sim$ Prep -1$)$

This article is protected by copyright. All rights reserved. 
summary(MRPrep)

\# Mean centered age

data.ES\$Age.PD<-data.ES\$Age.PD - mean(data.ES\$Age.PD, na.rm=T)

MRPDAge<-rma(yi $=y i, v i=v i$, data $=$ data.ES, mods $=\sim$ Age.PD)

summary(MRPDAge)

data.ES\$Age.Ct<-data.ES\$Age.Ct - mean(data.ES\$Age.Ct, na.rm=T)

MRCtAge $<-$ rma(yi $=$ yi, vi $=v i$, data $=$ data.ES, mods $=\sim$ Age.Ct $)$

summary(MRCtAge)

\# Difference in age

MRDiffAge $<-$ rma(yi $=$ yi, vi $=$ vi, data $=$ data.ES, mods $=\sim$ DiffAge $)$

summary(MRDiffAge)

\# Difference in PMI

MRDiffPMI<-rma(yi = yi, vi = vi, data $=$ data.ES, mods= $\sim$ DiffPMI)

summary(MRDiffPMI)

\# Difference in SR

MRDiffPercMale<-rma(yi $=$ yi, vi $=$ vi, data $=$ data.ES, mods $=\sim$ DiffPercMale $)$

summary(MRDiffPercMale)

\# Mean centered disease duration

data.ES\$Duration<-data.ES\$Duration - mean(data.ES\$Duration, na.rm=T)

MRDuration<-rma(yi $=$ yi, vi $=v i$, data $=$ data.ES, mods $=\sim$ Duration)

summary(MRDuration)

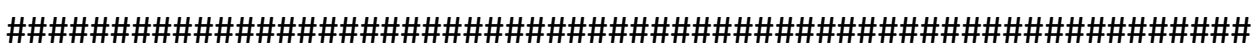

\#\#\#\#\#\#\#\#\#\#\# Meta-analyses For SN Zn data \#\#\#\#\#\#\#\#\#\#\#\#\#\#\#\#

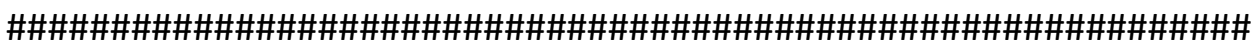

\# Deletes any potentially old info hanging around

$\mathrm{rm}(\operatorname{list}=\mathrm{Is}())$

\# Set the working directory

setwd("/Users/siangenoud/Desktop/Forests")

\# Install if needs be on the first time - intall.packages("metafor") and intall.packages("plyr")

\# Load packages we need

library("metafor")

library("plyr")

\# Load dataset

data<-read.csv("Zn SN quality order.csv")

This article is protected by copyright. All rights reserved. 
\# Check the data looks good

head(data)

\# Calculate the effect size ("SMD" - Hedges d) using escalc function

\#slab function labels studies

data.ES<-escalc(measure = "SMD", n1i = PD.cases, $n 2 \mathrm{i}=$ Ct.cases, $\mathrm{m} 1 \mathrm{i}=\mathrm{PD} . \mathrm{Zn}, \mathrm{m} 2 \mathrm{i}=\mathrm{Ct} . \mathrm{Zn}$, sd1 $\mathrm{i}=$ PD.Zn.SD, sd2i = Ct.Zn.SD, data = data, slab=paste(Author, Year, sep=", "))

\# Fit the meta analysis for all data

MA1<-rma(yi = yi, vi = vi, data $=$ data.ES)

summary(MA1)

\# Fit subsets for high and moderate quality studies

res.nL <- rma(yi=yi, vi=vi, data=data.ES, subset=(Quality=="High" | Quality == "Moderate"), method="REML")

summary(res.nL)

res.h $<-$ rma(yi=yi, vi=vi, data=data.ES, subset=(Quality=="High"), method="REML")

summary(res.h)

res. $m<-$ rma(yi=yi, vi=vi, data=data.ES, subset=(Quality=="Moderate"), method="REML")

summary(res.m)

\#Publication bias test

regtest(MA1)

\#Check for missing studies

trimfill(MA1)

\#Funnel plot

funnel(MA1)

\#\#\#\#\#\#\#\#\#\#\#\#\#\#\#\#\#\#\#\# Meta-regressions \#\#\#\#\#\#\#\#\#\#\#\#\#\#\#\#\#\#

\#\#\#\#Meta-regression analysis for effect of technique

\# This first line tells you the order - by default $\mathrm{R}$ will put the first one of these categories as the intercept group

sort(unique(data.ES\$TTechnique))

\# Here you change the intercept, by changing base $=x$ at the end - here I changed it to the second in the list above

\# Overall effect of analytical technique

contrasts(data.ES\$Technique)<-contr.treatment(levels(data.ES\$Technique), base=2)

MRTechnique<-rma(yi = yi, vi $=$ vi, data $=$ data.ES, mods $=\sim$ Technique-1)

summary(MRTechnique)

\# Overall effect of tissue prep

MRPrep<-rma(yi $=$ yi, vi $=v i$, data $=$ data.ES, mods $=\sim$ Prep -1$)$

summary(MRPrep)

This article is protected by copyright. All rights reserved. 


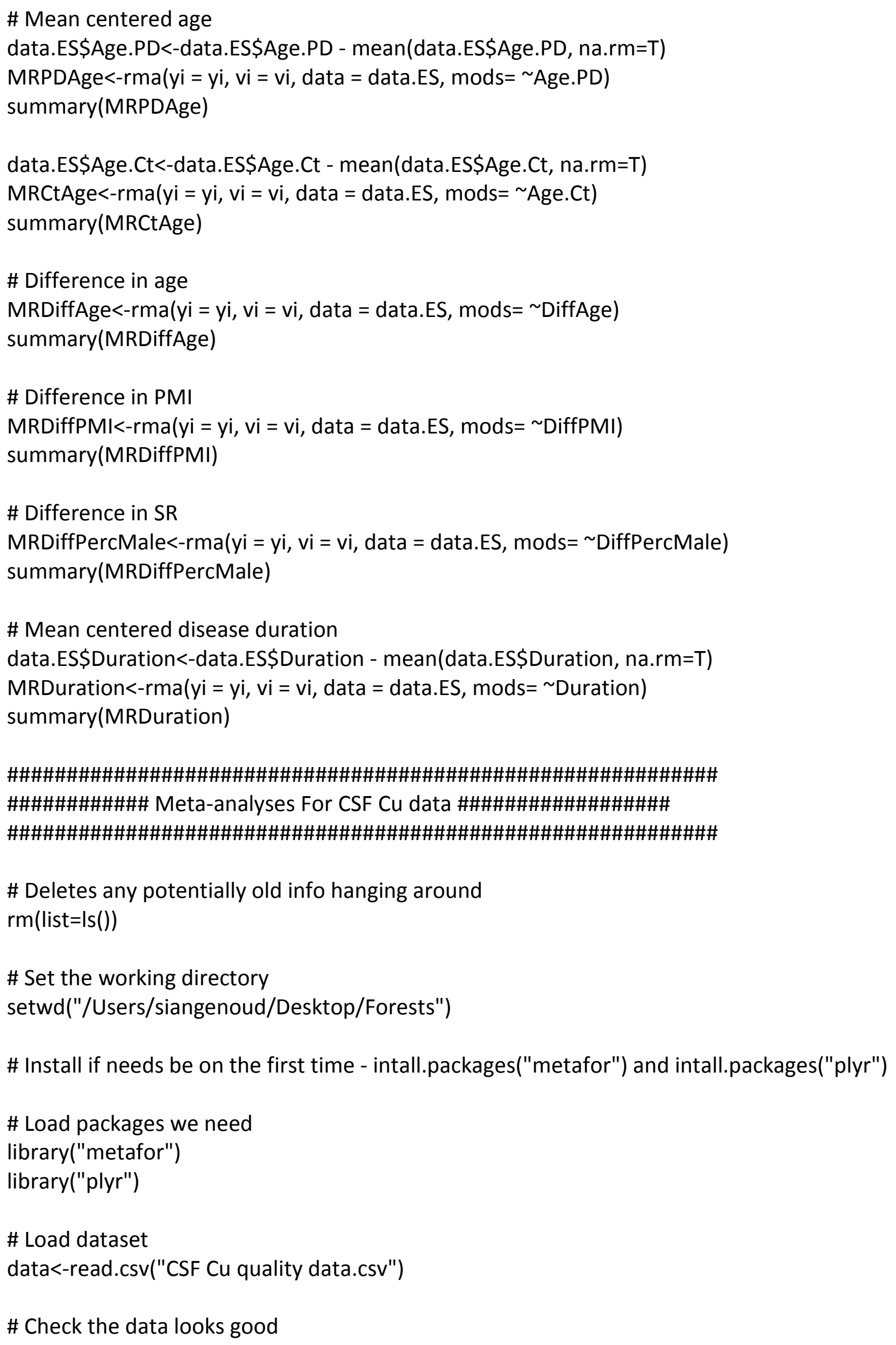


head(data)

\# Calculate the effect size ("SMD" - Hedges d) using escalc function

\#slab function labels studies

data.ES<-escalc(measure = "SMD", n1i = PD.cases, $\mathrm{n} 2 \mathrm{i}=$ Ct.cases, $\mathrm{m} 1 \mathrm{i}=\mathrm{PD} . \mathrm{Cu}, \mathrm{m} 2 \mathrm{i}=\mathrm{Ct} . \mathrm{Cu}$, sd1 $\mathrm{i}=$ PD.Cu.SD, sd2i = Ct.Cu.SD, data = data, slab=paste(Author, Year, sep=", "))

\# Fit the meta analysis for all data

MA1<-rma(yi = yi, vi = vi, data = data.ES)

summary(MA1)

\# Fit subsets by quality

res.h <- rma(yi=yi, vi=vi, data=data.ES, subset=(Quality=="High"), method="REML")

summary(res.h)

res.m <- rma(yi=yi, vi=vi, data=data.ES, subset=(Quality=="Moderate"), method="REML")

summary(res.m)

\#Publication bias test

regtest(MA1)

\#Check for missing studies

trimfill(MA1)

\#Funnel plot

funnel(MA1)

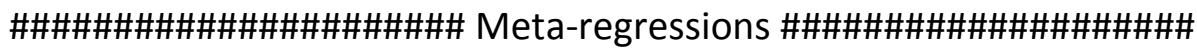

\#\#\#\#Meta-regression analysis for effect of technique

\# This first line tells you the order - by default $\mathrm{R}$ will put the first one of these categories as the intercept group

sort(unique(data.ES\$TTechnique))

\# Here you change the intercept, by changing base $=x$ at the end - here I changed it to the second in the list above

\# Overall effect of analytical technique

contrasts(data.ES\$Technique)<-contr.treatment(levels(data.ES\$Technique), base=2)

MRTechnique $<-r m a(y i=y i$, vi $=$ vi, data $=$ data.ES, mods $=\sim$ Technique -1$)$

summary(MRTechnique)

\# Mean centered age

data.ES\$Age.PD<-data.ES\$Age.PD - mean(data.ES\$Age.PD, na.rm=T)

MRPDAge<-rma(yi $=\mathrm{yi}, \mathrm{vi}=\mathrm{vi}$, data $=$ data.ES, mods $=\sim$ Age.PD $)$

summary(MRPDAge)

data.ES\$Age.Ct<-data.ES\$Age.Ct - mean(data.ES\$Age.Ct, na.rm=T)

This article is protected by copyright. All rights reserved. 
MRCtAge $<-r m a(y i=y i, v i=v i$, data $=$ data.ES, mods $=\sim$ Age.Ct $)$

summary(MRCtAge)

\# Difference in age

MRDiffAge $<-r m a(y i=y i$, vi $=v i$, data $=$ data.ES, mods $=\sim$ DiffAge $)$

summary(MRDiffAge)

\# Difference in SR

MRDiffPercMale $<-$ rma(yi $=$ yi, vi $=$ vi, data $=$ data.ES, mods $=\sim$ DiffPercMale $)$

summary(MRDiffPercMale)

\# Mean centered disease duration

data.ES\$Duration<-data.ES\$Duration - mean(data.ES\$Duration, na.rm=T)

MRDuration<-rma(yi $=$ yi, vi $=$ vi, data $=$ data.ES, mods $=\sim$ Duration $)$

summary(MRDuration)

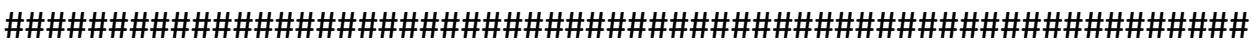

\#\#\#\#\#\#\#\#\#\#\# Meta-analyses For CSF Fe data \#\#\#\#\#\#\#\#\#\#\#\#\#\#\#\#

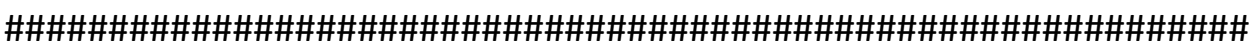

\# Deletes any potentially old info hanging around

$\mathrm{rm}(\operatorname{list}=\mathrm{Is}())$

\# Set the working directory

setwd("/Users/siangenoud/Desktop/Forests")

\# Install if needs be on the first time - intall.packages("metafor") and intall.packages("plyr")

\# Load packages we need

library("metafor")

library("plyr")

\# Load dataset

data<-read.csv("CSF Fe quality order.csv")

\# Check the data looks good

head(data)

\# Calculate the effect size ("SMD" - Hedges d) using escalc function

\#slab function labels studies

data.ES<-escalc(measure = "SMD", n1i = PD.cases, $n 2 i=$ Ct.cases, $\mathrm{m} 1 \mathrm{i}=$ PD.Fe, $\mathrm{m} 2 \mathrm{i}=\mathrm{Ct}$.Fe, sd1 $\mathrm{i}=$ PD.Fe.SD, sd2i = Ct.Fe.SD, data = data, slab=paste(Author, Year, sep=", "))

\# Fit the meta analysis for all data

MA1<-rma(yi = yi, vi = vi, data = data.ES)

summary(MA1)

This article is protected by copyright. All rights reserved. 
\# Fit subsets by quality

res.h <- rma(yi=yi, vi=vi, data=data.ES, subset=(Quality=="High"), method="REML") summary(res.h)

res.m <- rma(yi=yi, vi=vi, data=data.ES, subset=(Quality=="Moderate"), method="REML") summary(res.m)

\# No publicaiton bias as $\mathrm{k}<10$

\#\#\#\#\#\#\#\#\#\#\#\#\#\#\#\#\#\#\# Meta-regressions \#\#\#\#\#\#\#\#\#\#\#\#\#\#\#\#\#

\#\#\#\#Meta-regression analysis for effect of technique

\# This first line tells you the order - by default $\mathrm{R}$ will put the first one of these categories as the intercept group

sort(unique(data.ES\$Technique))

\# Here you change the intercept, by changing base $=x$ at the end - here I changed it to the second in the list above

\# Overall effect of analytical technique

contrasts(data.ES\$Technique)<-contr.treatment(levels(data.ES\$Technique), base=2)

MRTechnique<-rma(yi = yi, vi = vi, data = data.ES, mods $=\sim$ Technique-1)

summary(MRTechnique)

\# Mean centered age

data.ES\$Age.PD<-data.ES\$Age.PD - mean(data.ES\$Age.PD, na.rm=T)

MRPDAge $<-r m a(y i=y i, v i=v i$, data $=$ data.ES, mods $=\sim$ Age.PD $)$

summary(MRPDAge)

data.ES\$Age.Ct<-data.ES\$Age.Ct - mean(data.ES\$Age.Ct, na.rm=T)

MRCtAge<-rma(yi = yi, vi $=$ vi, data $=$ data.ES, mods $=\sim$ Age.Ct $)$

summary(MRCtAge)

\# Difference in age

MRDiffAge $<-r m a(y i=y i, v i=v i$, data $=$ data.ES, mods $=\sim$ DiffAge $)$

summary(MRDiffAge)

\# Difference in SR

MRDiffPercMale<-rma(yi $=$ yi, vi $=$ vi, data $=$ data.ES, mods $=\sim$ DiffPercMale $)$

summary(MRDiffPercMale)

\# Mean centered disease duration

data.ES\$Duration<-data.ES\$Duration - mean(data.ES\$Duration, na.rm=T)

MRDuration<-rma(yi $=\mathrm{yi}, \mathrm{vi}=\mathrm{vi}$, data $=$ data.ES, $\operatorname{mods}=\sim$ Duration $)$

summary(MRDuration)

This article is protected by copyright. All rights reserved. 


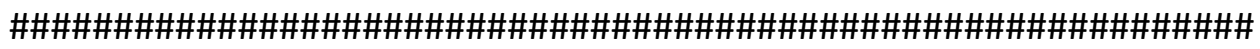 \\ \#\#\#\#\#\#\#\#\#\#\# Meta-analyses For CSF Zn data \#\#\#\#\#\#\#\#\#\#\#\#\#\#\#\#\#

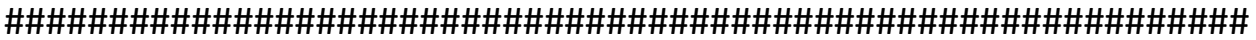 \\ \# Deletes any potentially old info hanging around \\ $\mathrm{rm}($ list $=\mathrm{Is}())$}

\# Set the working directory

setwd("/Users/siangenoud/Desktop/Forests")

\# Install if needs be on the first time - intall.packages("metafor") and intall.packages("plyr")

\# Load packages we need

library("metafor")

library("plyr")

\# Load dataset

data<-read.csv("CSF Zn quality order.csv")

\# Check the data looks good

head(data)

\# Calculate the effect size ("SMD" - Hedges d) using escalc function

\#slab function labels studies

data.ES<-escalc(measure = "SMD", n1i = PD.cases, $\mathrm{n} 2 \mathrm{i}=$ Ct.cases, $\mathrm{m} 1 \mathrm{i}=\mathrm{PD} . \mathrm{Zn}, \mathrm{m} 2 \mathrm{i}=\mathrm{Ct}$.Zn, sd1 $\mathrm{i}=$ PD.Zn.SD, sd2i = Ct.Zn.SD, data = data, slab=paste(Author, Year, sep=", "))

\# Fit the meta analysis for all data

MA1<-rma(yi = yi, vi = vi, data = data.ES)

summary(MA1)

\# Fit subsets by quality

res.h <- rma(yi=yi, vi=vi, data=data.ES, subset=(Quality=="High"), method="REML")

summary(res.h)

res.m <-rma(yi=yi, vi=vi, data=data.ES, subset=(Quality=="Moderate"), method="REML")

summary(res.m)

\# No publicaiton bias as $\mathrm{k}<10$

\#\#\#\#\#\#\#\#\#\#\#\#\#\#\#\#\#\#\# Meta-regressions \#\#\#\#\#\#\#\#\#\#\#\#\#\#\#\#\#\#

\#\#\#\#Meta-regression analysis for effect of technique

\# This first line tells you the order - by default $\mathrm{R}$ will put the first one of these categories as the intercept group

sort(unique(data.ES\$Technique))

This article is protected by copyright. All rights reserved. 
\# Here you change the intercept, by changing base $=x$ at the end - here I changed it to the second in the list above

\# Overall effect of analytical technique contrasts(data.ES\$Technique)<-contr.treatment(levels(data.ES\$Technique), base=2) MRTechnique<-rma(yi = yi, vi = vi, data $=$ data.ES, mods $=\sim$ Technique -1$)$ summary(MRTechnique)

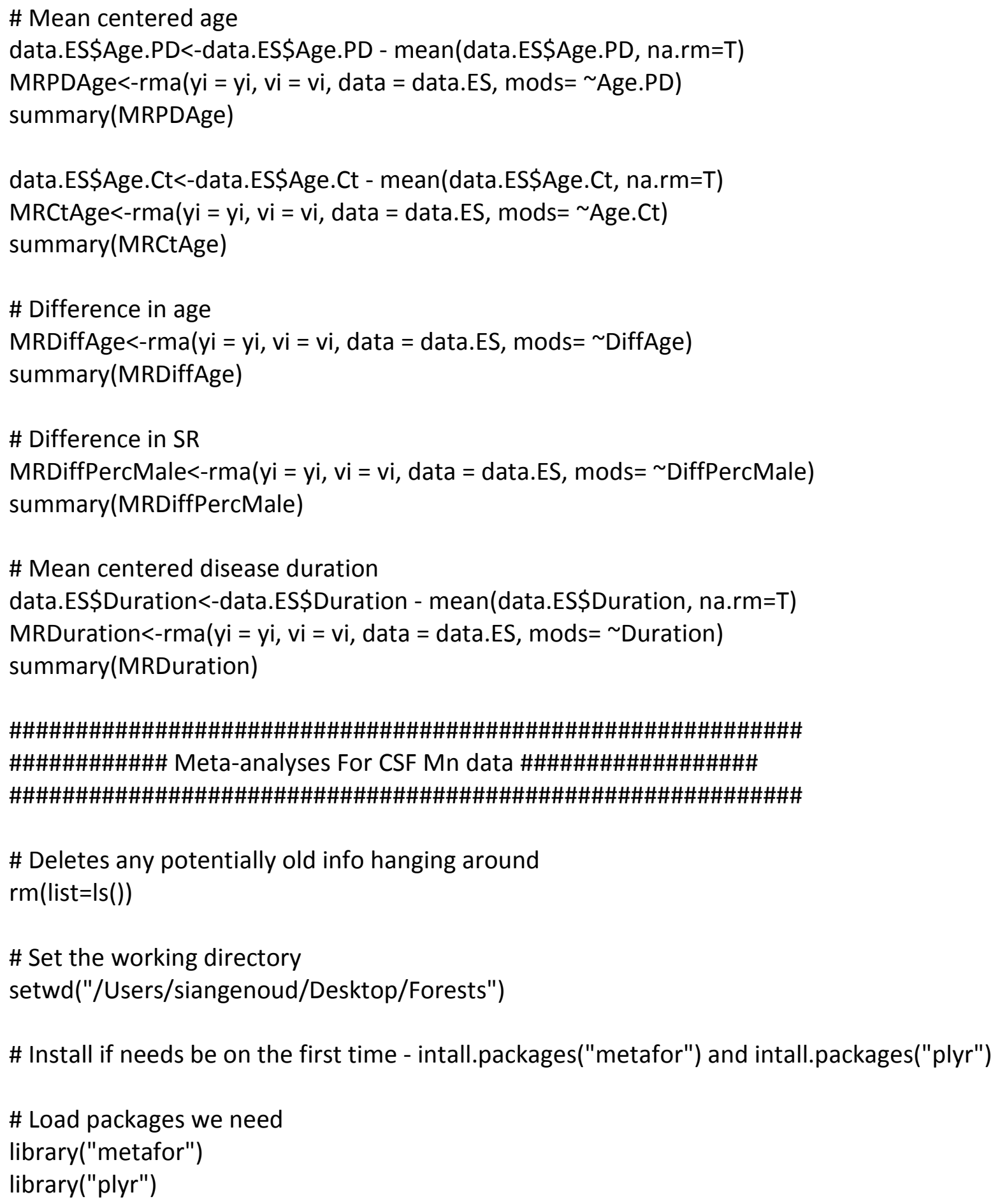




\section{\# Load dataset}

data<-read.csv("CSF Mn quality order.csv")

\# Check the data looks good

head(data)

\# Calculate the effect size ("SMD" - Hedges d) using escalc function

\#slab function labels studies

data.ES<-escalc(measure = "SMD", n1i = PD.cases, $\mathrm{n} 2 \mathrm{i}=$ Ct.cases, $\mathrm{m} 1 \mathrm{i}=\mathrm{PD} \cdot \mathrm{Mn}, \mathrm{m} 2 \mathrm{i}=\mathrm{Ct} . \mathrm{Mn}$, sd1 $\mathrm{i}=$ PD.Mn.SD, sd2i = Ct.Mn.SD, data = data, slab=paste(Author, Year, sep=", "))

\# Fit the meta analysis for all data

MA1<-rma(yi = yi, vi = vi, data = data.ES)

summary(MA1)

\# Fit subsets by quality

res.h <- rma(yi=yi, vi=vi, data=data.ES, subset=(Quality=="High"), method="REML")

summary(res.h)

res.m <- rma(yi=yi, vi=vi, data=data.ES, subset=(Quality=="Moderate"), method="REML")

summary(res.m)

\# No publicaiton bias as $\mathrm{k}<10$

\#\#\#\#\#\#\#\#\#\#\#\#\#\#\#\#\#\#\#\# Meta-regressions \#\#\#\#\#\#\#\#\#\#\#\#\#\#\#\#\#\#

\#\#\#\#Meta-regression analysis for effect of technique

\# This first line tells you the order - by default $\mathrm{R}$ will put the first one of these categories as

the intercept group

sort(unique(data.ES\$TTechnique))

\# Here you change the intercept, by changing base $=x$ at the end - here I changed it to the second in the list above

\# Overall effect of analytical technique

contrasts(data.ES\$Technique)<-contr.treatment(levels(data.ES\$TTechnique), base=2)

MRTechnique $<-r m a(y i=y i, v i=v i$, data $=$ data.ES, mods $=\sim$ Technique -1$)$

summary(MRTechnique)

\# Mean centered age

data.ES\$Age.PD<-data.ES\$Age.PD - mean(data.ES\$Age.PD, na.rm=T)

MRPDAge<-rma(yi $=\mathrm{yi}, \mathrm{vi}=\mathrm{vi}$, data $=$ data.ES, $\operatorname{mods}=\sim$ Age.PD $)$

summary(MRPDAge)

data.ES\$Age.Ct<-data.ES\$Age.Ct - mean(data.ES\$Age.Ct, na.rm=T)

MRCtAge<-rma(yi = yi, vi $=$ vi, data $=$ data. ES, mods $=\sim$ Age.Ct $)$

summary(MRCtAge)

This article is protected by copyright. All rights reserved. 


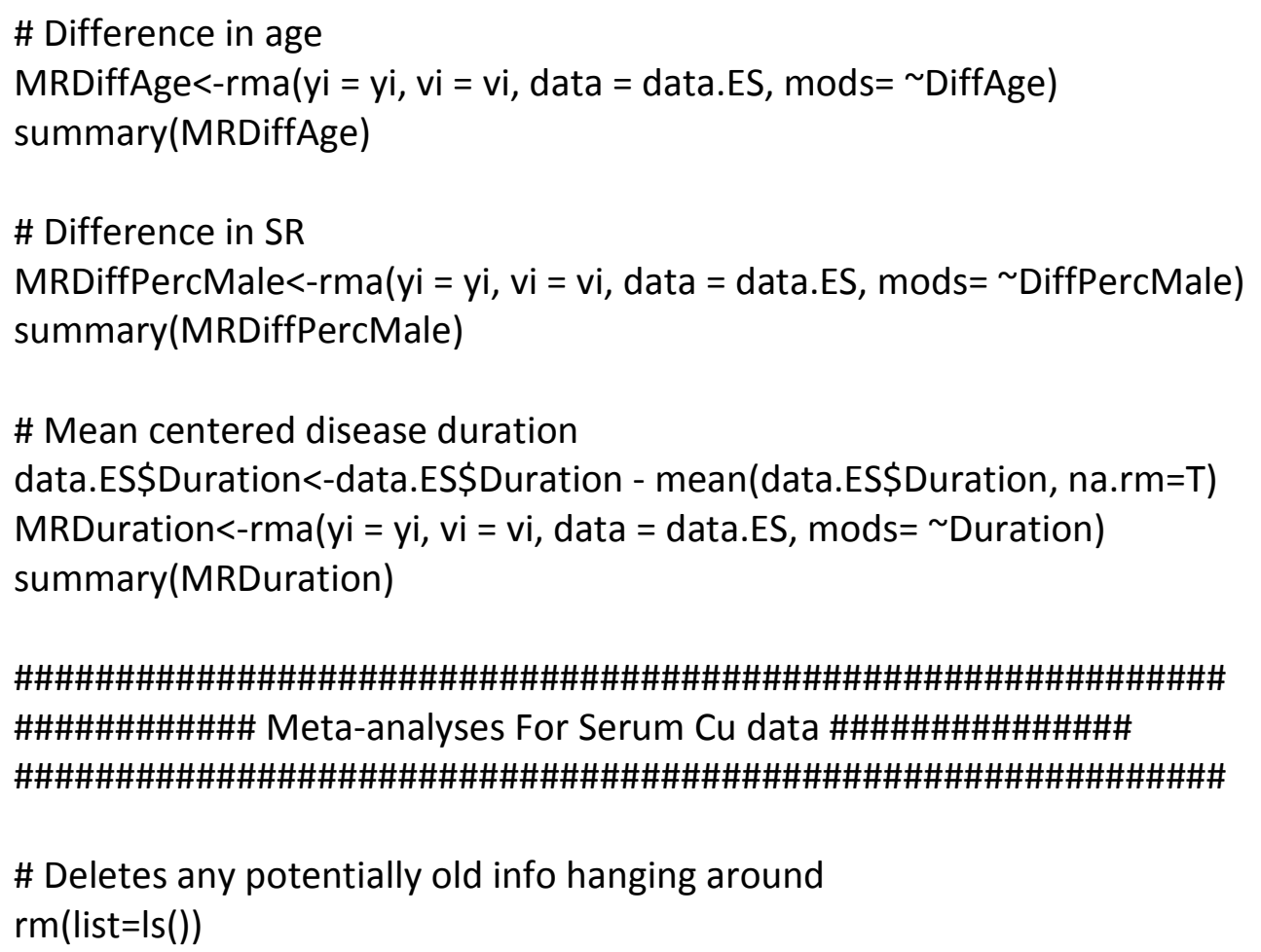

\# Fit the meta analysis for all data

MA1<-rma(yi = yi, vi = vi, data $=$ data.ES)

summary(MA1)

\# Fit subsets by quality

res.h <- rma(yi=yi, vi=vi, data=data.ES, subset=(Quality=="High"), method="REML") 
summary(res.h)

res.m $<-$ rma(yi=yi, vi=vi, data=data.ES, subset=(Quality=="Moderate"), method="REML") summary(res.m)

res.I <- rma(yi=yi, vi=vi, data=data.ES, subset=(Quality=="Limited"), method="REML")

summary(res.l)

\#Publication bias test

regtest(MA1)

\#Check for missing studies

trimfill(MA1)

\#Funnel plot

funnel(MA1)

\#\#\#\#\#\#\#\#\#\#\#\#\#\#\#\#\#\#\# Meta-regressions \#\#\#\#\#\#\#\#\#\#\#\#\#\#\#\#\#\#

\#\#\#\#Meta-regression analysis for effect of technique

\# This first line tells you the order - by default $\mathrm{R}$ will put the first one of these categories as the intercept group

sort(unique(data.ES\$TTechnique))

\# Here you change the intercept, by changing base $=x$ at the end - here I changed it to the second in the list above

\# Overall effect of analytical technique

contrasts(data.ES\$Technique)<-contr.treatment(levels(data.ES\$Technique), base=2)

MRTechnique<-rma(yi = yi, vi = vi, data $=$ data.ES, mods $=\sim$ Technique-1)

summary(MRTechnique)

\# Mean centered age

data.ES\$Age.PD<-data.ES\$Age.PD - mean(data.ES\$Age.PD, na.rm=T)

MRPDAge <-rma(yi = yi, vi $=v i$, data $=$ data.ES, mods $=\sim$ Age.PD $)$

summary(MRPDAge)

data.ES\$Age.Ct<-data.ES\$Age.Ct - mean(data.ES\$Age.Ct, na.rm=T)

MRCtAge $<-r m a(y i=y i, v i=v i$, data $=$ data. ES, mods $=\sim$ Age.Ct $)$

summary(MRCtAge)

\# Difference in age

MRDiffAge<-rma(yi $=$ yi, vi $=$ vi, data $=$ data.ES, mods $=\sim$ DiffAge $)$

summary(MRDiffAge)

\# Difference in PMI

MRDiffPMI<-rma(yi = yi, vi = vi, data = data.ES, mods= DiffPMI)

summary(MRDiffPMI)

\# Difference in SR

MRDiffPercMale $<-r m a(y i=y i$, vi $=$ vi, data $=$ data.ES, mods $=\sim$ DiffPercMale $)$

This article is protected by copyright. All rights reserved. 
summary(MRDiffPercMale)

\# Mean centered disease duration

data.ES\$Duration<-data.ES\$Duration - mean(data.ES\$Duration, na.rm=T)

MRDuration<-rma(yi $=\mathrm{yi}, \mathrm{vi}=\mathrm{vi}$, data $=$ data.ES, mods $=\sim$ Duration $)$

summary(MRDuration)

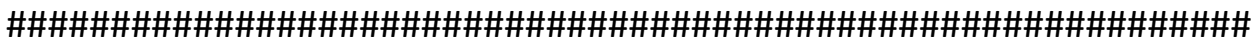

\#\#\#\#\#\#\#\#\#\#\# Meta-analyses For serum Fe data \#\#\#\#\#\#\#\#\#\#\#\#\#\#

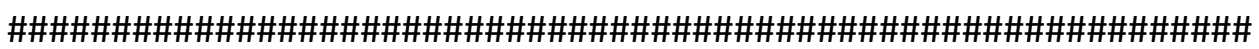

\# Deletes any potentially old info hanging around

$\mathrm{rm}($ list $=\mathrm{Is}())$

\# Set the working directory

setwd("/Users/siangenoud/Desktop/Forests")

\# Install if needs be on the first time - intall.packages("metafor") and intall.packages("plyr")

\# Load packages we need

library("metafor")

library("plyr")

\# Load dataset

data<-read.csv("Serum Fe quality order.csv")

\# Check the data looks good

head(data)

\# Calculate the effect size ("SMD" - Hedges d) using escalc function

\#slab function labels studies

data.ES<-escalc(measure = "SMD", n1 $\mathrm{i}=$ PD.cases, $\mathrm{n} 2 \mathrm{i}=$ Ct.cases, $\mathrm{m} 1 \mathrm{i}=\mathrm{PD} . \mathrm{Fe}, \mathrm{m} 2 \mathrm{i}=\mathrm{Ct}$.Fe, sd1 $\mathrm{i}=$ PD.Fe.SD, sd2i = Ct.Fe.SD, data = data, slab=paste(Author, Year, sep=", "))

\# Fit the meta analysis for all data

MA1<-rma(yi = yi, vi = vi, data $=$ data.ES)

summary(MA1)

\# Fit subsets by quality

res.nL <- rma(yi=yi, vi=vi, data=data.ES, subset=(Quality=="High" | Quality == "Moderate"), method="REML")

summary(res.nL)

res.h <- rma(yi=yi, vi=vi, data=data.ES, subset=(Quality=="High"), method="REML")

summary(res.h)

res.m <- rma(yi=yi, vi=vi, data=data.ES, subset=(Quality=="Moderate"), method="REML")

summary(res.m)

res.I <- rma(yi=yi, vi=vi, data=data.ES, subset=(Quality=="Limited"), method="REML")

This article is protected by copyright. All rights reserved. 
summary(res.l)

\#Publication bias test

regtest(MA1)

\#Check for missing studies

trimfill(MA1)

\#Funnel plot

funnel(MA1)

\#\#\#\#\#\#\#\#\#\#\#\#\#\#\#\#\#\#\# Meta-regressions \#\#\#\#\#\#\#\#\#\#\#\#\#\#\#\#\#

\#\#\#\#Meta-regression analysis for effect of technique

\# This first line tells you the order - by default $R$ will put the first one of these categories as the intercept group

sort(unique(data.ES\$TTechnique))

\# Here you change the intercept, by changing base $=x$ at the end - here I changed it to the second in the list above

\# Overall effect of analytical technique

contrasts(data.ES\$TTechnique)<-contr.treatment(levels(data.ES\$Technique), base=2)

MRTechnique<-rma(yi = yi, vi = vi, data = data.ES, mods= Technique-1)

summary(MRTechnique)

\# Mean centered age

data.ES\$Age.PD<-data.ES\$Age.PD - mean(data.ES\$Age.PD, na.rm=T)

MRPDAge<-rma(yi $=y i, v i=v i$, data $=$ data.ES, mods $=\sim$ Age.PD $)$

summary(MRPDAge)

data.ES\$Age.Ct<-data.ES\$Age.Ct - mean(data.ES\$Age.Ct, na.rm=T)

MRCtAge $<-$ rma $(y i=y i, v i=v i$, data $=$ data.ES, mods $=\sim$ Age.Ct $)$

summary(MRCtAge)

\# Difference in age

MRDiffAge $<-$ rma $(y i=y i$, vi $=v i$, data $=$ data.ES, mods $=\sim$ DiffAge $)$

summary(MRDiffAge)

\# Difference in SR

MRDiffPercMale<-rma(yi $=$ yi, vi $=v i$, data $=$ data.ES, mods $=\sim$ DiffPercMale $)$

summary(MRDiffPercMale)

\# Mean centered disease duration

data.ES\$Duration<-data.ES\$Duration - mean(data.ES\$Duration, na.rm=T)

MRDuration<-rma(yi = yi, vi = vi, data $=$ data.ES, mods $=\sim$ Duration)

summary(MRDuration)

\#\#\#\#\#\#\#\#\#\#\#\#\#\#\#\#\#\#\#\#\#\#\#\#\#\#\#\#\#\#\#\#\#\#\#\#\#\#\#\#\#\#\#\#\#\#\#\#\#\#\#\#\#

This article is protected by copyright. All rights reserved. 
\#\#\#\#\#\#\#\#\#\# Meta-analyses For serum Zn data \#\#\#\#\#\#\#\#\#\#\#\#\#\#

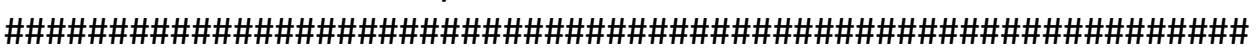

\# Deletes any potentially old info hanging around

$\mathrm{rm}($ list $=\mathrm{Is}())$

\# Set the working directory

setwd("/Users/siangenoud/Desktop/Forests")

\# Install if needs be on the first time - intall.packages("metafor") and intall.packages("plyr")

\# Load packages we need

library("metafor")

library("plyr")

\# Load dataset

data<-read.csv("Serum Zn quality order.csv")

\# Check the data looks good

head(data)

\# Calculate the effect size ("SMD" - Hedges d) using escalc function

\#slab function labels studies

data.ES<-escalc(measure = "SMD", n1i = PD.cases, $n 2 \mathrm{i}=$ Ct.cases, $\mathrm{m} 1 \mathrm{i}=\mathrm{PD} . \mathrm{Zn}, \mathrm{m} 2 \mathrm{i}=\mathrm{Ct} . \mathrm{Zn}$, sd1 $i=$ PD.Zn.SD, sd2i = Ct.Zn.SD, data = data, slab=paste(Author, Year, sep=", "))

\# Fit the meta analysis for all data

MA1<-rma(yi $=$ yi, vi $=$ vi, data $=$ data.ES)

summary(MA1)

\# Fit subsets by quality

res.nL <- rma(yi=yi, vi=vi, data=data.ES, subset=(Quality=="High" | Quality == "Moderate"), method="REML")

summary(res.nL)

res.h <- rma(yi=yi, vi=vi, data=data.ES, subset=(Quality=="High"), method="REML")

summary(res.h)

res.m <- rma(yi=yi, vi=vi, data=data.ES, subset=(Quality=="Moderate"), method="REML")

summary(res.m)

\#Publication bias test

regtest(MA1)

\#Check for missing studies

trimfill(MA1)

\#Funnel plot

funnel(MA1)

\#\#\#\#\#\#\#\#\#\#\#\#\#\#\#\#\#\#\# Meta-regressions \#\#\#\#\#\#\#\#\#\#\#\#\#\#\#\#\#\#

This article is protected by copyright. All rights reserved. 
\#\#\#\#Meta-regression analysis for effect of technique

\# This first line tells you the order - by default $\mathrm{R}$ will put the first one of these categories as the intercept group

sort(unique(data.ES\$TTechnique))

\# Here you change the intercept, by changing base $=x$ at the end - here I changed it to the second in the list above

\# Overall effect of analytical technique

contrasts(data.ES\$TTechnique)<-contr.treatment(levels(data.ES\$TTechnique), base=2)

MRTechnique $<-r m a(y i=y i, v i=v i$, data $=$ data.ES, mods $=\sim$ Technique -1$)$

summary(MRTechnique)

\# Mean centered age

data.ES\$Age.PD<-data.ES\$Age.PD - mean(data.ES\$Age.PD, na.rm=T)

MRPDAge<-rma(yi $=y i, v i=v i$, data $=$ data.ES, mods $=\sim$ Age.PD $)$

summary(MRPDAge)

data.ES\$Age.Ct<-data.ES\$Age.Ct - mean(data.ES\$Age.Ct, na.rm=T)

MRCtAge<-rma(yi $=y i, v i=v i$, data $=$ data. ES, mods $=\sim$ Age.Ct $)$

summary(MRCtAge)

\# Difference in age

MRDiffAge $<-r m a(y i=y i$, vi $=v i$, data $=$ data.ES, mods $=\sim$ DiffAge $)$

summary(MRDiffAge)

\# Difference in SR

MRDiffPercMale<-rma(yi $=$ yi, vi $=$ vi, data $=$ data.ES, mods $=\sim$ DiffPercMale)

summary(MRDiffPercMale)

\# Mean centered disease duration

data.ES\$Duration<-data.ES\$Duration - mean(data.ES\$Duration, na.rm=T)

MRDuration $<-r m a(y i=y i$, vi $=v i$, data $=$ data.ES, $\operatorname{mods}=\sim$ Duration $)$

summary(MRDuration)

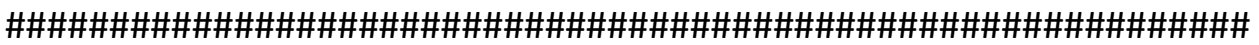

\#\#\#\#\#\#\#\#\#\#\# Meta-analyses For serum Mn data \#\#\#\#\#\#\#\#\#\#\#\#\#

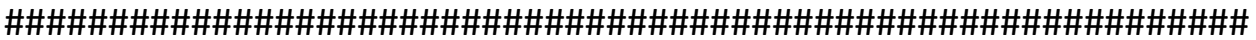

\# Deletes any potentially old info hanging around

$\mathrm{rm}(\operatorname{list}=\mathrm{Is}())$

\# Set the working directory

setwd("/Users/siangenoud/Desktop/Forests")

\# Install if needs be on the first time - intall.packages("metafor") and intall.packages("plyr")

This article is protected by copyright. All rights reserved. 
\# Load packages we need

library("metafor")

library("plyr")

\# Load dataset

data<-read.csv("Serum Mn quality order.csv")

\# Check the data looks good

head(data)

\# Calculate the effect size ("SMD" - Hedges d) using escalc function

\#slab function labels studies

data.ES<-escalc(measure = "SMD", n1i = PD.cases, $\mathrm{n} 2 \mathrm{i}=$ Ct.cases, $\mathrm{m} 1 \mathrm{i}=\mathrm{PD} \cdot \mathrm{Mn}, \mathrm{m} 2 \mathrm{i}=\mathrm{Ct} . \mathrm{Mn}$, $\mathrm{sd} 1 \mathrm{i}=\mathrm{PD} . \mathrm{Mn} . \mathrm{SD}, \mathrm{sd} 2 \mathrm{i}=\mathrm{Ct} . \mathrm{Mn} . \mathrm{SD}$, data = data, slab=paste(Author, Year, sep=", "))

\# Fit the meta analysis for all data

MA1<-rma(yi = yi, vi = vi, data = data.ES)

summary(MA1)

\# Fit subsets by quality

res. $n L<-$ rma(yi=yi, vi=vi, data=data.ES, subset=(Quality=="High" | Quality == "Moderate"), method="REML")

summary(res.nL)

res.h <- rma(yi=yi, vi=vi, data=data.ES, subset=(Quality=="High"), method="REML")

summary(res.h)

res. $m<-$ rma(yi=yi, vi=vi, data=data.ES, subset=(Quality=="Moderate"), method="REML")

summary(res.m)

\#Publication bias test

regtest(MA1)

\#Check for missing studies

trimfill(MA1)

\#Funnel plot

funnel(MA1)

\#\#\#\#\#\#\#\#\#\#\#\#\#\#\#\#\#\#\#\# Meta-regressions \#\#\#\#\#\#\#\#\#\#\#\#\#\#\#\#\#\#

\#\#\#\#Meta-regression analysis for effect of technique

\# This first line tells you the order - by default $\mathrm{R}$ will put the first one of these categories as

the intercept group

sort(unique(data.ES\$TTechnique))

\# Here you change the intercept, by changing base $=x$ at the end - here I changed it to the second in the list above

\# Overall effect of analytical technique

This article is protected by copyright. All rights reserved. 
contrasts(data.ES\$TTechnique)<-contr.treatment(levels(data.ES\$Technique), base=2)

MRTechnique $<-$ rma $(\mathrm{yi}=\mathrm{yi}$, vi $=\mathrm{vi}$, data $=$ data.ES, mods $=\sim$ Technique -1$)$

summary(MRTechnique)

\# Mean centered age

data.ES\$Age.PD<-data.ES\$Age.PD - mean(data.ES\$Age.PD, na.rm=T)

MRPDAge $<-r m a(y i=y i, v i=v i$, data $=$ data.ES, $\operatorname{mods}=\sim$ Age.PD $)$

summary(MRPDAge)

data.ES\$Age.Ct<-data.ES\$Age.Ct - mean(data.ES\$Age.Ct, na.rm=T)

MRCtAge<-rma(yi = yi, vi = vi, data $=$ data. $E S$, mods $=\sim$ Age.Ct $)$

summary(MRCtAge)

\# Difference in age

MRDiffAge $<-r m a(y i=y i$, vi $=$ vi, data $=$ data.ES, mods $=\sim$ DiffAge $)$

summary(MRDiffAge)

\# Difference in SR

MRDiffPercMale<-rma(yi $=$ yi, vi $=$ vi, data $=$ data.ES, mods $=\sim$ DiffPercMale)

summary(MRDiffPercMale)

\# Mean centered disease duration

data.ES\$Duration<-data.ES\$Duration - mean(data.ES\$Duration, na.rm=T)

MRDuration<-rma(yi $=\mathrm{yi}, \mathrm{vi}=\mathrm{vi}$, data $=$ data.ES, $\operatorname{mods}=\sim$ Duration $)$

summary(MRDuration)

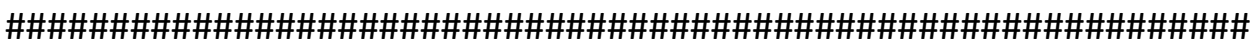

\#\#\#\#\#\#\#\#\#\#\# Meta-analyses For Plasma Cu data \#\#\#\#\#\#\#\#\#\#\#\#\#

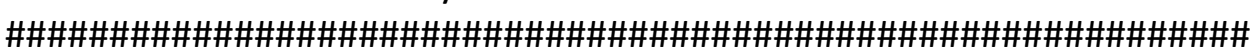

\# Deletes any potentially old info hanging around

rm(list=ls())

\# Set the working directory

setwd("/Users/siangenoud/Desktop/Forests")

\# Install if needs be on the first time - intall.packages("metafor") and intall.packages("plyr")

\# Load packages we need

library("metafor")

library("plyr")

\# Load dataset

data<-read.csv("Plasma Cu quality order.csv")

\# Check the data looks good

head(data)

This article is protected by copyright. All rights reserved. 
\# Calculate the effect size ("SMD" - Hedges d) using escalc function

\#slab function labels studies

data.ES<-escalc(measure = "SMD", n1i = PD.cases, $\mathrm{n} 2 \mathrm{i}=$ Ct.cases, $\mathrm{m} 1 \mathrm{i}=\mathrm{PD} \cdot \mathrm{Cu}, \mathrm{m} 2 \mathrm{i}=\mathrm{Ct} . \mathrm{Cu}$, sd1i = PD.Cu.SD, sd2i = Ct.Cu.SD, data = data, slab=paste(Author, Year, sep=", "))

\# Fit the meta analysis for all data

MA1<-rma(yi = yi, vi = vi, data = data.ES)

summary(MA1)

\# Fit subsets by quality

res. $\mathrm{nL}<-$ rma(yi=yi, vi=vi, data=data.ES, subset=(Quality == "Moderate" |

Quality=="Limited"), method="REML")

summary(res.nL)

res. $m<-$ rma(yi=yi, vi=vi, data=data.ES, subset=(Quality=="Moderate"), method="REML") summary(res.m)

res.I <- rma(yi=yi, vi=vi, data=data.ES, subset=(Quality=="Limited"), method="REML")

summary(res.l)

\#Publication bias test

regtest(MA1)

\#Check for missing studies

trimfill(MA1)

\#Funnel plot

funnel(MA1)

\#\#\#\#\#\#\#\#\#\#\#\#\#\#\#\#\#\#\# Meta-regressions \#\#\#\#\#\#\#\#\#\#\#\#\#\#\#\#\#\#

\#\#\#\#Meta-regression analysis for effect of technique

\# This first line tells you the order - by default $\mathrm{R}$ will put the first one of these categories as the intercept group

sort(unique(data.ES\$TTechnique))

\# Here you change the intercept, by changing base $=x$ at the end - here I changed it to the second in the list above

\# Overall effect of analytical technique

contrasts(data.ES\$Technique)<-contr.treatment(levels(data.ES\$Technique), base=2)

MRTechnique<-rma(yi = yi, vi = vi, data $=$ data.ES, mods $=\sim$ Technique-1)

summary(MRTechnique)

\# Mean centered age

data.ES\$Age.PD<-data.ES\$Age.PD - mean(data.ES\$Age.PD, na.rm=T)

MRPDAge $<-r m a(y i=y i, v i=v i$, data $=$ data.ES, mods= $\sim$ Age.PD $)$

summary(MRPDAge)

data.ES\$Age.Ct<-data.ES\$Age.Ct - mean(data.ES\$Age.Ct, na.rm=T)

This article is protected by copyright. All rights reserved. 
MRCtAge $<-r m a(y i=y i, v i=v i$, data $=$ data.ES, mods $=\sim$ Age.Ct $)$

summary(MRCtAge)

\# Difference in age

MRDiffAge $<-r m a(y i=y i, v i=v i$, data $=$ data.ES, mods $=\sim$ DiffAge $)$

summary(MRDiffAge)

\# Difference in SR

MRDiffPercMale<-rma(yi $=$ yi, vi $=v i$, data $=$ data.ES, mods $=\sim$ DiffPercMale $)$

summary(MRDiffPercMale)

\# Not enough studies to mean centered disease duration

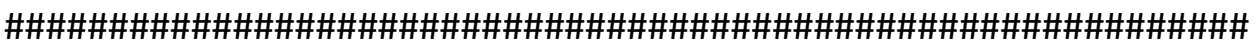

\#\#\#\#\#\#\#\#\#\#\# Meta-analyses For Plasma Fe data \#\#\#\#\#\#\#\#\#\#\#\#\#\#

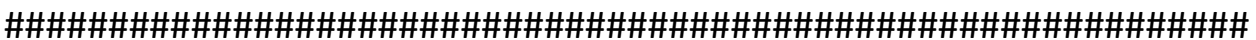

\# Deletes any potentially old info hanging around

$\mathrm{rm}(\operatorname{list}=\mathrm{Is}())$

\# Set the working directory

setwd("/Users/siangenoud/Desktop/Forests")

\# Install if needs be on the first time - intall.packages("metafor") and intall.packages("plyr")

\# Load packages we need

library("metafor")

library("plyr")

\# Load dataset

data<-read.csv("Plasma Fe quality order.csv")

\# Check the data looks good

head(data)

\# Calculate the effect size ("SMD" - Hedges d) using escalc function

\#slab function labels studies

data.ES<-escalc(measure = "SMD", n1 i = PD.cases, $\mathrm{n} 2 \mathrm{i}=$ Ct.cases, $\mathrm{m} 1 \mathrm{i}=\mathrm{PD} . \mathrm{Fe}, \mathrm{m} 2 \mathrm{i}=\mathrm{Ct}$.Fe, sd $1 \mathrm{i}=$ PD.Fe.SD, sd2i = Ct.Fe.SD, data = data, slab=paste(Author, Year, sep=", "))

\# Fit the meta analysis for all data

MA1<-rma(yi = yi, vi = vi, data = data.ES)

summary(MA1)

\# Fit subsets by quality

res. $m<-$ rma(yi=yi, vi=vi, data=data.ES, subset=(Quality=="Moderate"), method="REML") summary(res.m)

This article is protected by copyright. All rights reserved. 
\#Publication bias test

regtest(MA1)

\#Check for missing studies

trimfill(MA1)

\#Funnel plot

funnel(MA1)

\#\#\#\#\#\#\#\#\#\#\#\#\#\#\#\#\#\#\# Meta-regressions \#\#\#\#\#\#\#\#\#\#\#\#\#\#\#\#\#\#

\#\#\#\#Meta-regression analysis for effect of technique

\# This first line tells you the order - by default $\mathrm{R}$ will put the first one of these categories as the intercept group

sort(unique(data.ES\$Technique))

\# Not enough techniques to analyse overall effect of analytical technique

\# Not enough studies to analyse effect of Age, Sex ratio or disease duration

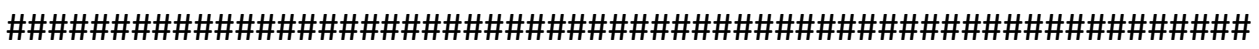

\#\#\#\#\#\#\#\#\#\#\# Meta-analyses For Plasma Zn data \#\#\#\#\#\#\#\#\#\#\#\#\#\#

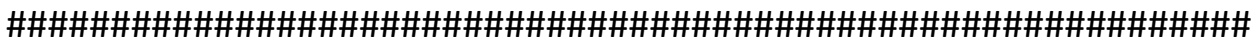

\# Deletes any potentially old info hanging around

$\mathrm{rm}(\operatorname{list}=\mathrm{Is}())$

\# Set the working directory

setwd("/Users/siangenoud/Desktop/Forests")

\# Install if needs be on the first time - intall.packages("metafor") and intall.packages("plyr")

\# Load packages we need

library("metafor")

library("plyr")

\# Load dataset

data<-read.csv("Plasma Zn quality order.csv")

\# Check the data looks good

head(data)

\# Calculate the effect size ("SMD" - Hedges d) using escalc function

\#slab function labels studies

data.ES<-escalc(measure = "SMD", n1i = PD.cases, $n 2 \mathrm{i}=$ Ct.cases, $\mathrm{m} 1 \mathrm{i}=\mathrm{PD} . \mathrm{Zn}, \mathrm{m} 2 \mathrm{i}=\mathrm{Ct} . \mathrm{Zn}$, sd1 $\mathrm{i}=$ PD.Zn.SD, sd2i = Ct.Zn.SD, data = data, slab=paste(Author, Year, sep=", "))

This article is protected by copyright. All rights reserved. 
\# Fit the meta analysis for all data

MA1<-rma(yi = yi, vi = vi, data = data.ES)

summary(MA1)

\# Fit subsets by quality

res.m <- rma(yi=yi, vi=vi, data=data.ES, subset=(Quality=="Moderate"), method="REML")

summary(res.m)

res.I <- rma(yi=yi, vi=vi, data=data.ES, subset=(Quality=="Limited"), method="REML")

summary(res.l)

\#Publication bias test

regtest(MA1)

\#Check for missing studies

trimfill(MA1)

\#Funnel plot

funnel(MA1)

\#\#\#\#\#\#\#\#\#\#\#\#\#\#\#\#\#\#\# Meta-regressions \#\#\#\#\#\#\#\#\#\#\#\#\#\#\#\#\#

\#\#\#\#Meta-regression analysis for effect of technique

\# This first line tells you the order - by default $\mathrm{R}$ will put the first one of these categories as

the intercept group

sort(unique(data.ES\$̦Technique))

\# Not enough techniques to analyse overall effect of analytical technique

\# Not enough studies to analyse effect of Age, Sex ratio or disease duration

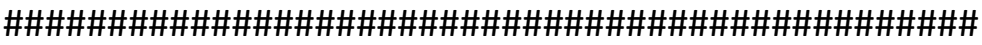 \\ \#\#\#\#\# REMA Regression of Brain vs Biofluids \#\#\#

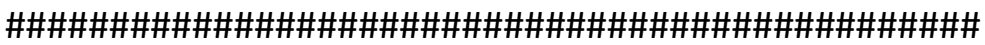

\# Deletes any potentially old info hanging around

$\mathrm{rm}($ list $=\mathrm{Is}())$

\# Set the working directory

setwd("/Users/siangenoud/Desktop/Forests")

\# Intall if needs be on the first time - intall.packages("metafor")

\# Load packages we need

library("metafor")

\# Load dataset

data<-read.csv("Combined Cu data 16.2.19.csv")

This article is protected by copyright. All rights reserved. 
\# Check the data looks good

head(data)

data.ES<-escalc(measure = "SMD", n1i = noPD, n2i = noCt, m1i =Cu.PD, m2i = Cu.Ct, sd1 i = SD.PD, sd2i = SD.Ct, data = data, slab=paste(Author, Year, sep=", "))

\# Again aded na.rm

data.ES\$Age.PD<-data.ES\$Age.PD - mean(data.ES\$Age.PD, na.rm=T)

\# Here you change the intercept, by changing base $=x$ at the end - here I changed it to the second in the list above sort(unique(data.ES\$Tissue))

contrasts(data.ES\$Tissue)<-contr.treatment(levels(data.ES\$Tissue), base=4)

\# OK effect of PD on CU levels is significantly different in SN than all bio fluids \# Effect of tissue

MRTissue $<-r m a(y i=y i, v i=v i$, data $=$ data.ES, mods $=\sim$ Tissue $)$

summary(MRTissue)

\# Agaein there are differences in age, so could this affect the results mean(data.ES\$Age.PD[which(data.ES\$Tissue $==$ "SN")], na.rm=T)

mean(data.ES\$Age.PD[which(data.ES\$TTissue == "Serum")], na.rm=T)

MRTissueAge $<-r m a(y i=y i, v i=v i$, data $=$ data.ES, mods $=\sim$ Tissue+Age.PD)

summary(MRTissueAge)

\# No the differences remain - no real effect of age

\#\#\#\#\#\# Repeat for the Fe data \#\#\#\#\#\#\#

\# Deletes any potentially old info hanging around $\mathrm{rm}($ list=Is ()$)$

\# Set the working directory setwd("/Users/siangenoud/Desktop/Forests")

\# Intall if needs be on the first time - intall.packages("metafor")

\# Load packages we need

library("metafor")

\# Load dataset

data<-read.csv("Fe combined tissue data 16.2.19.csv")

\# Check the data looks good

head(data)

This article is protected by copyright. All rights reserved. 
data.ES<-escalc(measure = "SMD", n1i = noPD, n2i = noCt, m1i $=$ Fe.PD, $m 2 \mathrm{i}=\mathrm{Fe} . \mathrm{Ct}$, sd1i $=$ SD.PD, sd2i = SD.Ct, data = data, slab=paste(Author, Year, sep=", "))

\# This was causing a problem because of the NAs in the dataset - I added na.rm=T at the end and htis fixed the issue

data.ES\$Age.PD<-data.ES\$Age.PD - mean(data.ES\$Age.PD, na.rm=T)

\# Here you change the intercept, by changing base $=x$ at the end - here I changed it to the second in the list above sort(unique(data.ES\$Tissue))

contrasts(data.ES\$Tissue)<-contr.treatment(levels(data.ES\$Tissue), base=4)

\# Effect of tissue

MRTissue $<-$ rma $(y i=y i$, vi $=$ vi, data $=$ data.ES, mods $=\sim$ Tissue $)$

summary(MRTissue)

\# Could results be affected by age - after all by the time brain tissue is collected PD is more advanced

mean(data.ES\$Age.PD[which(data.ES\$Tissue == "SN")], na.rm=T)

mean(data.ES\$Age.PD[which(data.ES\$Tissue $==$ "Serum")], na.rm=T)

MRTissueAge<-rma(yi = yi, vi $=$ vi, data $=$ data.ES, mods $=\sim$ Tissue+Age.PD)

summary(MRTissueAge)

\#\#\#\#\#\# Repeat for the Zn data \#\#\#\#\#\#

\# Deletes any potentially old info hanging around

$\mathrm{rm}(\operatorname{list}=\mathrm{Is}())$

\# Set the working directory

setwd("/Users/siangenoud/Desktop/Forests")

\# Intall if needs be on the first time - intall.packages("metafor")

\# Load packages we need

library("metafor")

\# Load dataset

data<-read.csv("Combined Zn data 18.2.19.csv")

\# Check the data looks good

head(data)

data.ES<-escalc(measure = "SMD", n1i = noPD, n2i = noCt, $m 1 \mathrm{i}=\mathrm{Zn} \cdot \mathrm{PD}, \mathrm{m} 2 \mathrm{i}=\mathrm{Zn} \cdot \mathrm{Ct}, \mathrm{sd} 1 \mathrm{i}=$ SD.PD, sd2i = SD.Ct, data = data, slab=paste(Author, Year, sep=", "))

This article is protected by copyright. All rights reserved. 
\# This was causing a problem because of the NAs in the dataset $-I$ added na.rm=T at the end and htis fixed the issue

data.ES\$Age.PD<-data.ES\$Age.PD - mean(data.ES\$Age.PD, na.rm=T)

\# Here you change the intercept, by changing base $=x$ at the end - here I changed it to the second in the list above

sort(unique(data.ES\$Tissue))

contrasts(data.ES\$Tissue)<-contr.treatment(levels(data.ES\$Tissue), base=4)

\# Effect of tissue

MRTissue $<-r m a(y i=y i, v i=v i$, data $=$ data.ES, mods $=\sim$ Tissue $)$

summary(MRTissue)

\# Could results be affected by age - after all by the time brain tissue is collected PD is more advanced

mean(data.ES\$Age.PD[which(data.ES\$Tissue $==$ "SN")], na.rm=T)

mean(data.ES\$Age.PD[which(data.ES\$Tissue == "Serum")], na.rm=T)

MRTissueAge<-rma(yi $=\mathrm{yi}, \mathrm{vi}=\mathrm{vi}$, data $=$ data.ES, mods $=\sim$ Tissue+Age.PD $)$

summary(MRTissueAge)

This article is protected by copyright. All rights reserved. 


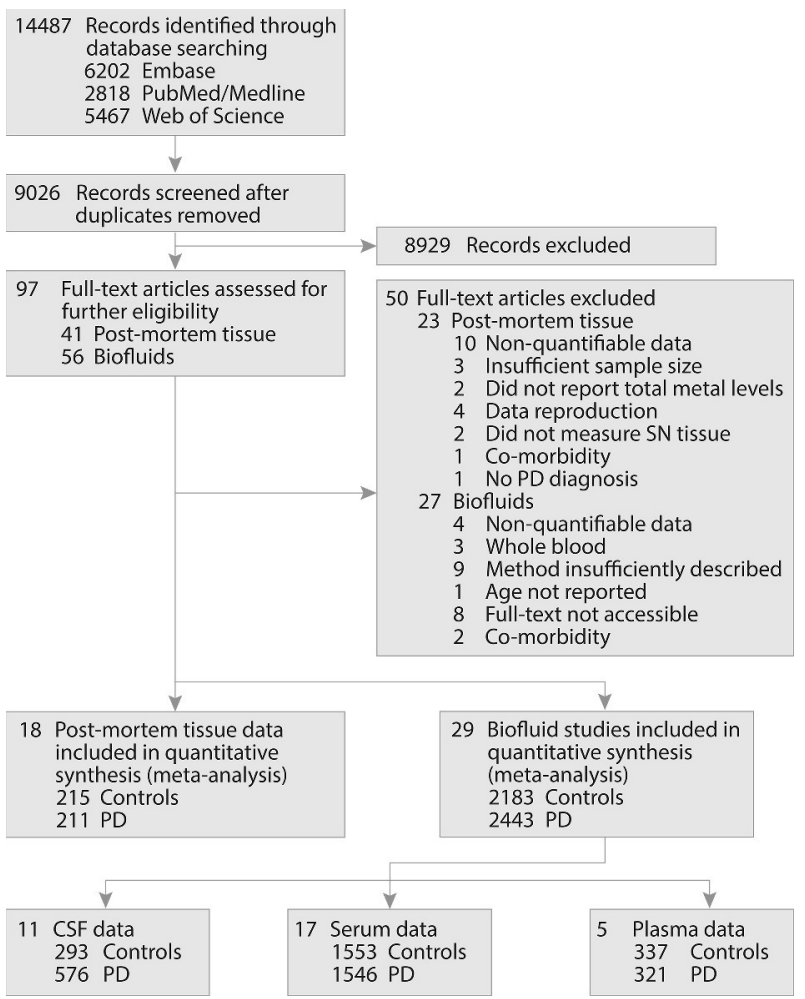

mds_27947_figure_1.eps 


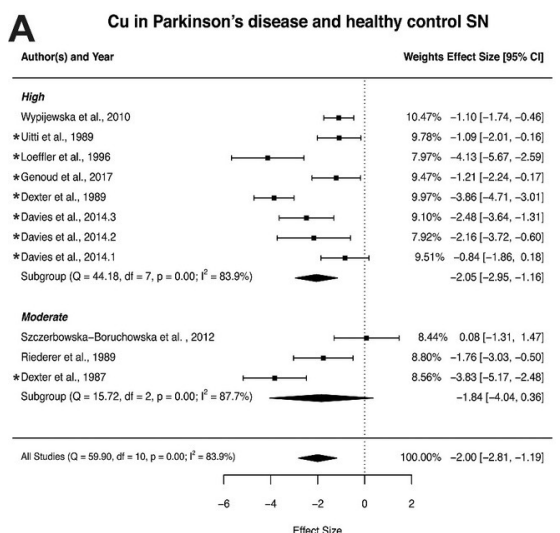

B Fe in Parkinson's disease and healthy control SN

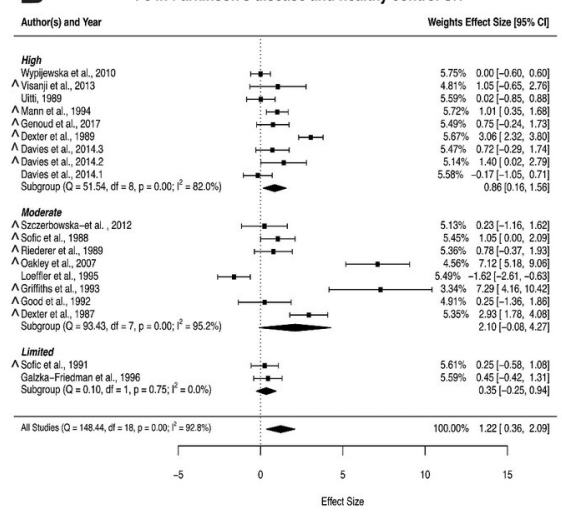

C $\quad \mathrm{n}$ in Parkinson's disease and healthy control SN

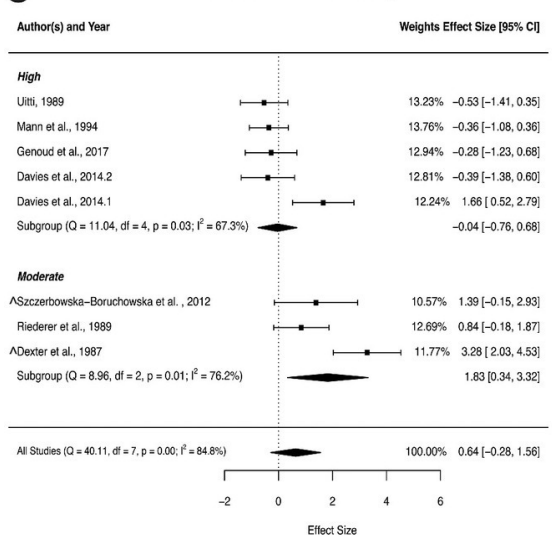

mds_27947_figure_2.eps 
A

Serum Cu in Parkinson's disease and healthy controls

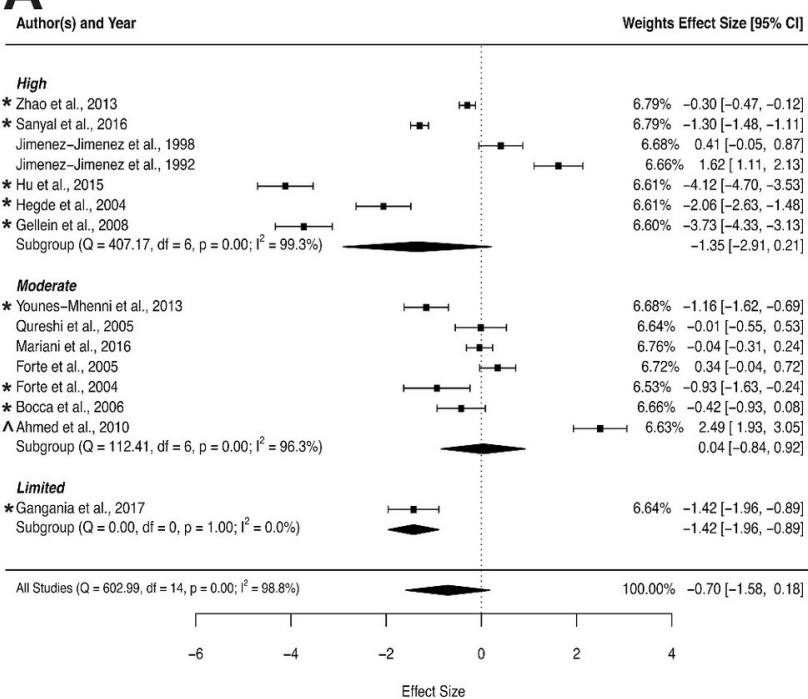

C Serum Fe in Parkinson's disease and healthy controls

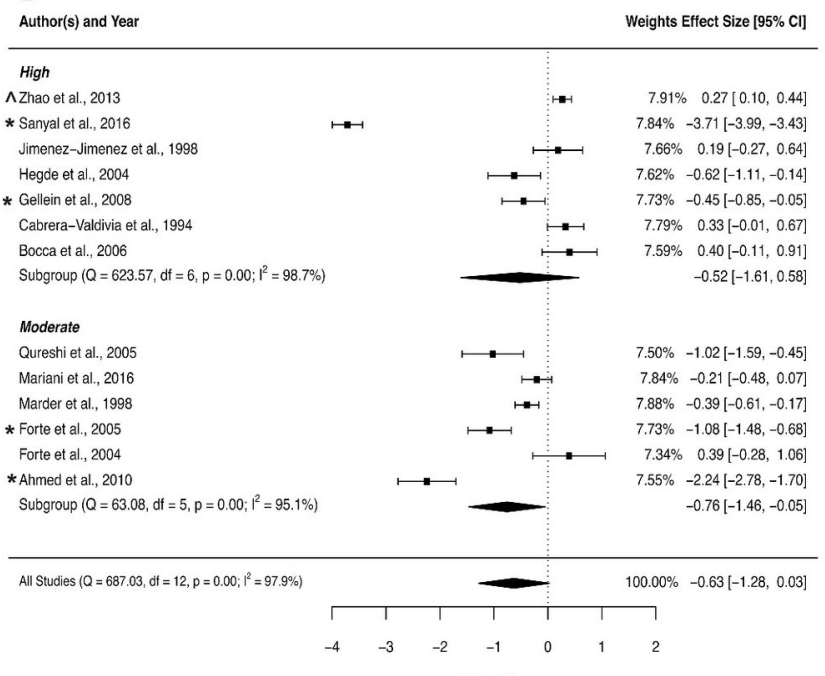

B Serum Fe in Parkinson's disease and healthy controls

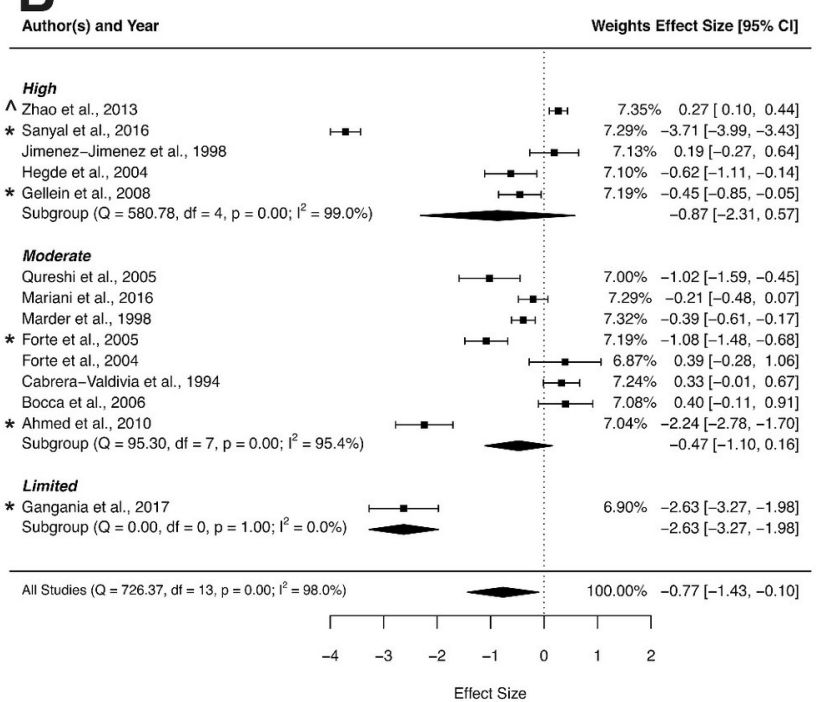

Serum Zn in Parkinson's disease and healthy controls

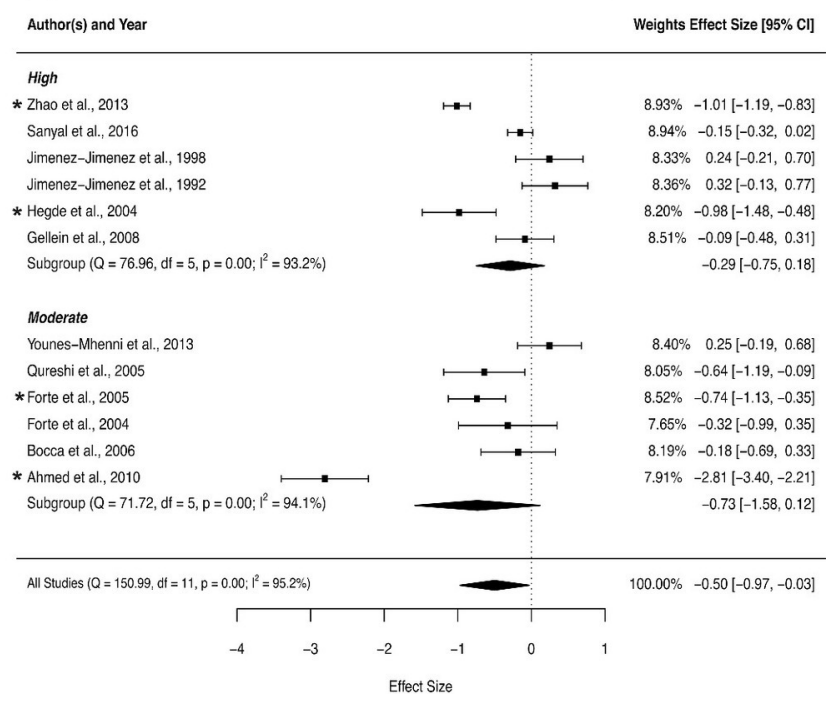

\begin{tabular}{l} 
Serum Mn in Parkinson's disease and healthy controls \\
Author(s) and Year \\
\hline
\end{tabular}

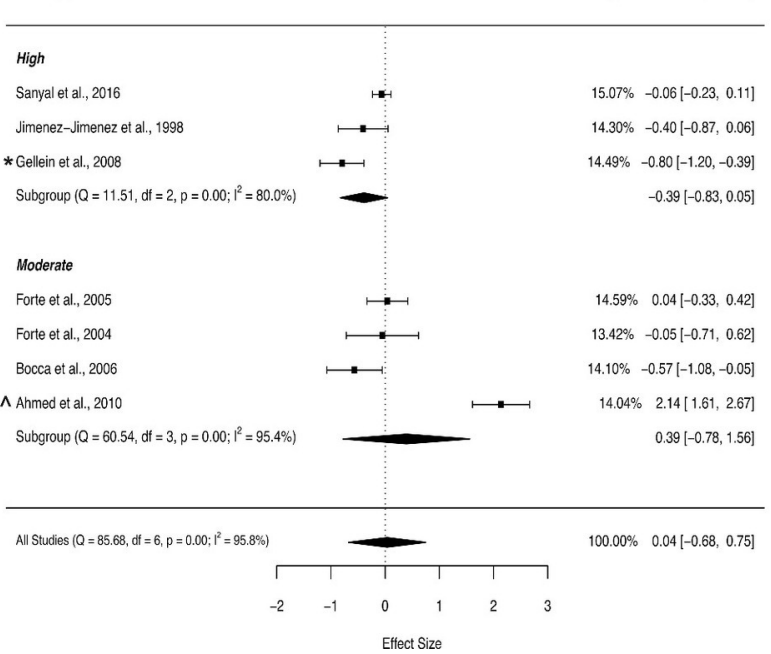

mds_27947_figure_3.eps

This article is protected by copyright. All rights reserved. 
A

CSF Cu in Parkinson's disease and healthy controls

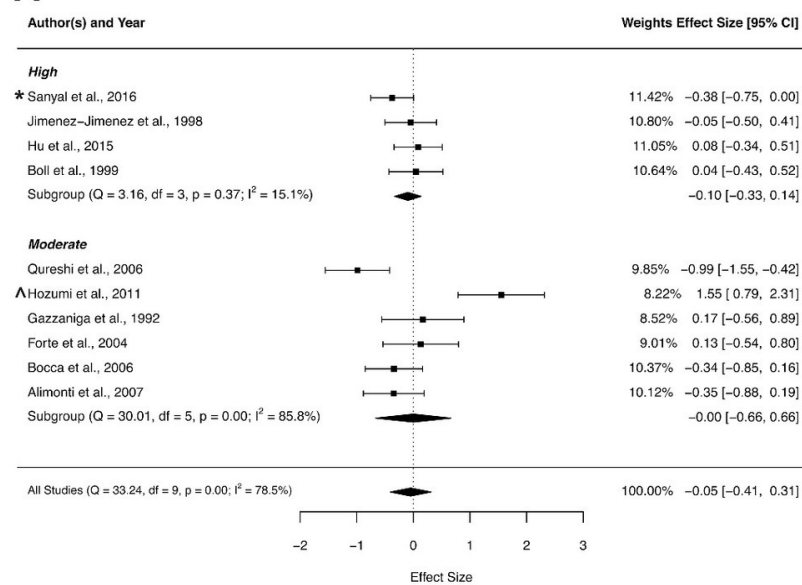

C

CSF $\mathrm{Zn}$ in Parkinson's disease and healthy controls

Author(s) and Year

\begin{tabular}{l|l}
\hline High & \\
* Sanyal et al., 2016 & $14.96 \%-0.44[-0.82,-0.06]$
\end{tabular}

* Jimenez-Jimenez et al., 1998

Subgroup $\left(Q=0.42, d f=1, p=051: i^{2}=0.0 \%\right)$

$-0.52[-0.82,-0.23]$

Moderate

* Qureshi et al., 2006

AHozumi et al, 2011

Forte et al., 2004

Bocca et al., 2006

Alimonti et al., 2007

Subgroup $\left(Q=54.72\right.$, df $=4, p=0.00 ;\left.\right|^{2}=94.3 \%$

AI Studios $\left(Q=5.50 .50\right.$, df $\left.=6, P=0.00 ; P^{2}=93.1 \%\right)$
B CSF Fe in Parkinson's disease and healthy controls

Author(s) and Year

\begin{tabular}{l:ll}
\hline High & \\
\hline
\end{tabular}

* Sanyal et al., $2016 \quad 11.57 \%-0.45[-0.83,-0.07]$ Jimenez-Jimenez et al., 1998 Subgroup $\left(Q=0.45, d f=1, p=0.50 ; I^{2}=0.0 \%\right)$

Moderate

$\wedge$ Wang et al. 2016

^Qureshi et al., 2006

Hozumi et al., 2011

Hozumi et al., 2011

* Gazzaniga et al., 1922

* Forte et al., 2004

* Bocca et al., 2006

* Bocca et al, 2006
*Alimonti et al, 2007
Subgroup $\left(Q=95.07\right.$, df =6, $\left.p=0.00 ;\left.\right|^{2}=94.7 \%\right)$

$0.11[-0.86,1.08]$

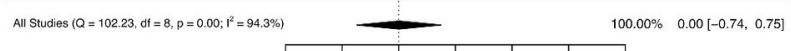

All Studies $\left\{\mathrm{Q}=102.23\right.$, of $\left.=8, \mathrm{p}=0.00 ; \mathrm{I}^{2}=94.3 \%\right\}$

Effect Size

D

CSF Mn in Parkinson's disease and healthy controls

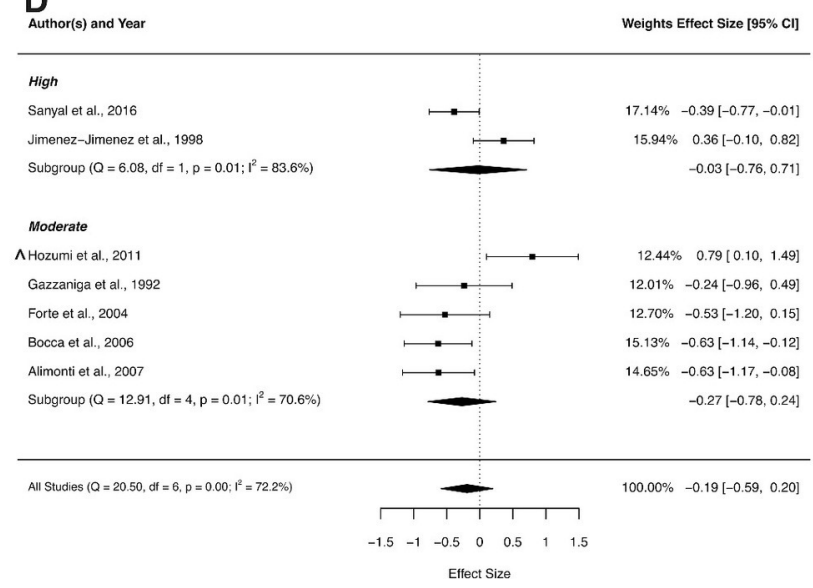

mds_27947_figure_4.eps 


\section{COPYRIGHT TRANSFER AGREEMENT}

Date: $\quad$ 8th October 2019

Contributor name: _ Kay Lorraine Double

Contributor address: 94-100 Mallet St, Camperdown, Sydney, NSW, Australia

Manuscript number: $\frac{\text { MDS-19-0841 }}{\text { Meta-analysis of copper and iron in }}$

Re: Manuscript entitled: Parkinson's disease brain and biofluids (the "Contribution")

for publication in: Movement Disorders (the "Journal")

Published by Wiley on behalf of The International Parkinson and Movement Disorder Society (the "Owner")

Dear Contributor(s):

Thank you for submitting your Contribution for publication. In order to expedite the editing and publishing process and enable the Owner to disseminate your Contribution to the fullest extent, we need to have this Copyright Transfer Agreement executed. If the Contribution is not accepted for publication, or if the Contribution is subsequently rejected, this Agreement shall be null and void. Publication cannot proceed without a signed copy of this Agreement.

\section{A. COPYRIGHT}

The Contributor assigns to the Owner, during the full term of copyright and any extensions or renewals, all copyright in and to the Contribution, and all rights therein, including but not limited to the right to reproduce, publish, republish, transmit, sell, transfer, distribute, and otherwise use the Contribution in whole or in part in electronic and print editions of the Journal and in derivative works throughout the world, in all languages and in all media of expression now known or later developed, and to license or permit others to do so.

\section{B. RETAINED RIGHTS}

Notwithstanding the above, the Contributor or, if applicable, the Contributor's employer, retains all proprietary rights other than copyright, such as patent rights, in any process, procedure or article of manufacture described in the Contribution. This reservation of rights does not affect or limit the rights assigned to Owner in Section A.

\section{PERMITTED USES BY CONTRIBUTOR}

1. License. The Owner grants to Contributor a non-exclusive, non-transferable and limited license to reproduce and distribute copies of the print or electronic "preprints" of the unpublished Contribution, in the original form submitted to the Journal prior to the peer review process, solely to colleagues within the Contributor's nonprofit organization or educational institution. The Contributor shall make no more than 100 printed copies of the preprints in any calendar year. Such preprints may be posted as electronic files on the Contributor's own personal website, on the Contributor's internal intranet at Contributor's nonprofit organization or educational institution, or on a secure external website at the Contributor's nonprofit organization or educational institution, provided that access is limited to employees and/or students at Contributor's non-profit organization or educational institution. Contributor shall not charge a fee for any 
preprints, and Contributor's use under this Section C shall not be for any commercial purpose, or for any systematic external distribution (e.g., posting on a listserve, public website, database connected to a public access server, or automated delivery system). The license grant in this Section does not apply to for-profit corporations, and any proposed use outside of the scope of this Section C must be pre-approved in writing by the Owner. The rights granted to Contributor under this Section $\mathrm{C}$ do not include reproduction, distribution or any other use of rating scales, videos or other audiovisual materials associated with the Contribution.

2. Required Citation. Prior to publication, the Contributor must provide full credit and acknowledgement of the Journal in all preprints in the following format: This is a preprint of an article accepted for publication in [Journal Title], Copyright (C) [year] The International Parkinson and Movement Disorder Society. After publication, the Contributor must provide a citation to the Journal in all preprints in the following format: This is a preprint of an article that was published in [Journal title]: (Title of Article, Contributor, Journal Title and Volume/ Issue, Copyright (C) [year] The International Parkinson and Movement Disorder Society). An electronic link must be provided to the Journal's website, located at http://www.interscience.Wiley.com. The Contributor agrees not to update the preprint or replace it with the published version of the Contribution.

3. Accepted Version. Re-use of the accepted and peer-reviewed (but not the final typeset published) version of the Contribution (the "Accepted Version") is not permitted under this Agreement. There are separate arrangements with certain funding agencies governing reuse of the Accepted Version. Additional terms apply if the Contributor receives or received funding from these agencies. The details of those relationships, and other offerings allowing open web use, are set forth at the following website: http://www.wiley.com/go/ funderstatement.

4. Additional Terms for Certain Funders. Certain funders, including the NIH, members of the Research Councils UK (RCUK) and Wellcome Trust require deposit of the Accepted Version in a public repository after an embargo period. Details of funding arrangements are set out at the following website: http://www.wiley.com/go/funderstatement. Additional terms may be applicable. Please contact the production editor for the journal at MDSprod@wiley.com if you have additional funding requirements.

If any Contributor receiving funds from applicable sources does not choose the Owner's OnlineOpen option, the Contributor will be allowed to self-archive by depositing the Accepted Version in a public repository after the following applicable embargo period has expired, subject to further conditions imposed by the RCUK:

a. $\quad 12$ months from first publication online of the final published version of the Contribution for research funded by members of the Research Councils UK (RCUK) other than The Economic and Social Research Council (ESRC) and the Arts and Humanities Research Council (AHRC); or

b. 24 months from first publication online of the final published version of the Contribution for research funded by ESRC or AHRC.

5. Additional Terms for Certain Institutions. Wiley has arrangements with certain educational institutions to permit the deposit of the Accepted Version in the institutional repository after an embargo period. Details of such arrangements are set out at the following website: http://olabout.wiley.com/WileyCDA/Section/id-406074.html . Additional terms may be applicable.

If any Contributor affiliated with these applicable educational institutions does not choose the Owner's OnlineOpen option, the Contributor will be allowed to self-archive by depositing the Accepted Version in the educational institution's repository after the following applicable embargo period has expired. See the following website for details: http://olabout.wiley.com/WileyCDA/Section/id-817011.html. 


\section{CONTRIBUTIONS OWNED BY EMPLOYER}

If the Contribution was written by the Contributor in the course of the Contributor's employment (as a "work-made-for-hire" in the course of employment), the Contribution is owned by the company/institution which must execute this Agreement (in addition to the Contributor's signature). In such case, the company/institution hereby assigns to the Owner, during the full term of copyright, all copyright in and to the Contribution for the full term of copyright throughout the world as specified in Section A above.

\section{E. GOVERNMENT CONTRACTS}

In the case of a Contribution prepared under U.S. Government contract or grant, the U.S. Government may reproduce, without charge, all or portions of the Contribution and may authorize others to do so, for official U.S. Government purposes only, if the U.S. Government contract or grant so requires. (U.S. Government, U.K. Government, and other government employees: see notes at end.)

\section{F. CONTRIBUTOR'S REPRESENTATIONS}

The Contributor represents that the Contribution is the Contributor's original work, all individuals identified as Contributors actually contributed to the Contribution, and all individuals who contributed are included. The Contribution is submitted only to this Journal and has not been published before. (If excerpts from copyrighted works owned by third parties are included, the Contributor will obtain written permission from the copyright owners for all uses as set forth in the Journal's Instructions for Contributors, and show credit to the sources in the Contribution.) The Contributor also warrants that the Contribution contains no libelous or unlawful statements, does not infringe upon the rights (including without limitation the copyright, patent or trademark rights) or the privacy of others, or contain material or instructions that might cause harm or injury. Upon request, Contributor will provide the data or will cooperating fully in obtaining and providing the data on which the Contribution is based for examination by the editors or their assignees.

\section{G. FINANCIAL DISCLOSURES}

The Contributor certifies that his/her financial and material support for this research and work, regardless of date, is clearly identified on Exhibit A to this Agreement. The Contributor has also identified on Exhibit A, all other support unrelated to this research, covering the past year from the date of submission (e.g., grants, advisory boards, employment, consultancies, contracts, honoraria, royalties, expert testimony, partnerships, or stock ownership in medically-related fields).

\section{H. VIDEO AND PHOTOGRAPHY CONSENT}

In the event that the Contribution includes, discloses or incorporates any content (including, without limitation, any video clip or photograph) which identifies any individual patient(s) ("patient identifiable content"), the Contributor obtained from such patient(s) written consent to such inclusion, disclosure or incorporation and that this consent fully complies with all legal requirements, including without limitation, all of the requirements of the laws of the jurisdiction(s) to which the patient(s) and the patient(s)' physician are subject, including the United States Health Insurance Portability and Accountability Act of 1996 ("HIPAA") if applicable. The Contributor hereby certifies that, if the patient consent form is in a language other than English, such consent form meets all of the requirements set forth in the Instructions to Authors. In addition, the Contributor hereby confirms that he/she obtained from patient(s) written consent to use the patient identifiable content in both print and online (i.e., internet/web-based) publication formats. The Contributor further certifies that the person executing any such patient consent form, to the best of his/her knowledge, had legal capacity under applicable law to execute the form on behalf of the patient. 


\section{ACKNOWLEDGEMENTS}

The Contributor should obtain written permission from all individuals named in the acknowledgement since readers may infer their endorsement of data and conclusions. The Contributor certifies that all individuals named in the acknowledgement section have provided written permission to be named.

\section{J. MISCELLANEOUS}

This Agreement may be amended or modified only in a writing executed by both parties. The waiver or failure of any party to exercise any rights under this Agreement shall not be deemed a waiver or other limitation of any other right or any future right. This Agreement shall inure to the benefit of, and shall be binding upon, the parties, their respective successors and permitted assigns. This Agreement may be executed in two (2) or more counterparts, each of which shall be an original and all of which taken together shall constitute one and the same agreement. Executed copies of this Agreement may be delivered by facsimile transmission, pdf/email or other comparable electronic means. If for any reason any provision of this Agreement shall be deemed by a court of competent jurisdiction to be legally invalid or unenforceable, the validity, legality and enforceability of the remainder of this Agreement shall not be affected and such provision shall be deemed modified to the minimum extent necessary to make such provision consistent with applicable law and, in its modified form, such provision shall then be enforceable and enforced. The parties agree to do such further acts and to execute and deliver such additional agreements and instruments from time to time as either may at any time reasonably request in order to assure and confirm unto such requesting party the rights, powers and remedies conferred in the Agreement. This Agreement, including any exhibits attached hereto, contains the entire agreement and understanding of the parties with respect to the subject matter hereof, and supersedes all prior agreements, negotiations, representations and proposals, written and oral, relating thereto.

\section{All Contributors must sign below. Contributors must check one box except that NIH grantees should check both Contributor-owned work and the NIH grantee box. If your Contribution was written during the course of employment, your employer must also sign where indicated.}

Please send your original completed and signed forms by fax or email a scanned copy to the Journal production editor. For production editor contact details please visit the Journal's online author guidelines. Do not send in hard copies of these forms.

\section{[ X ] Contributor-owned work}

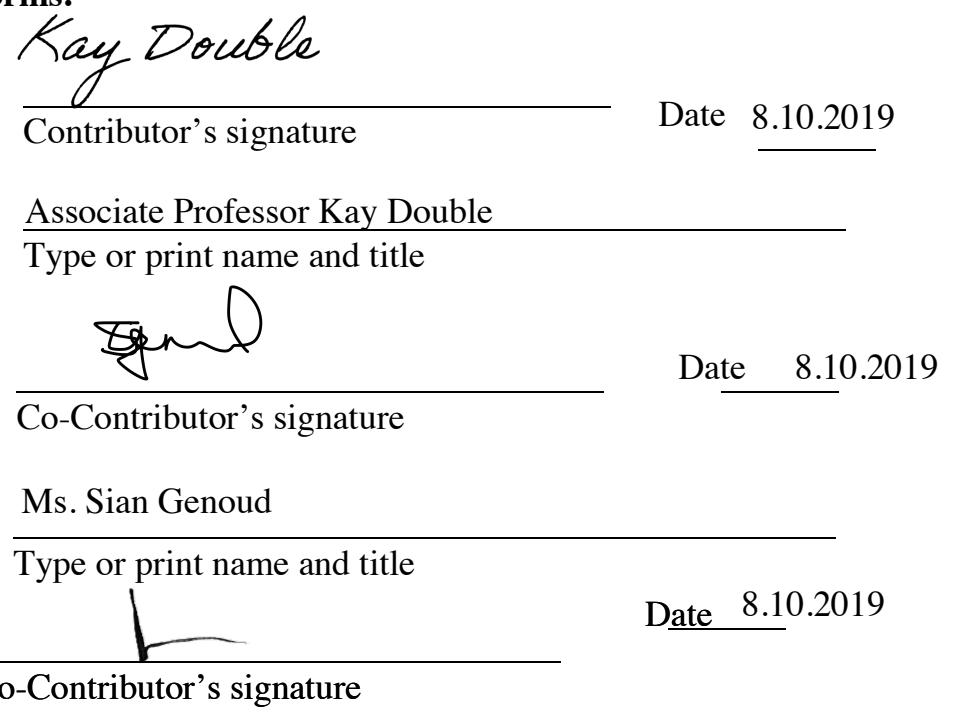

Associate Professor Dominic Hare

Type or print name and title 


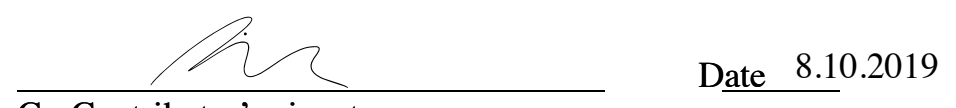

\section{Co-Contributor's signature}

Dr. Alistair Senior

Type or print name and title

[_ ] Company/Institution-owned

Work (made-for-hire in the $\overline{\text { Company or Institution (Employer-for-Hire) }} \overline{\text { Date }}$

Course of employment)

$\overline{\text { Authorized signature of Employer }} \quad \overline{\text { Date }}$

$\overline{\text { Contributor's signature }} \quad \overline{\text { Date }}$

Type or print name and title

\section{ATTACH ADDITIONAL SIGNATURE PAGES AS NECESSARY}

\section{[__ U.S. Government work}

Note to U.S. Government Employees

A contribution prepared by a U.S. federal government employee as part of the employee's official duties, or which is an official U.S. Government publication, is called a "U.S. Government work", and is in the public domain in the United States. In such case, Paragraph A.1 will not apply but the Contributor must type his/her name (in the Contributor's signature line) above. Contributor acknowledges that the Contribution will be published in the United States and other countries. If the Contribution was not prepared as part of the employee's duties or is not an official U.S. Government publication, it is not a U.S. Government work.

\section{[__ ] U.K. Government work (Crown Copyright)}

Note to U.K. Government Employees

The rights in a contribution prepared by an employee of a UK government department, agency or other Crown body as part of his/her official duties, or which is an official government publication, belong to the Crown. Contributors must ensure they comply with departmental regulations and submit the appropriate authorisation to publish. If your status as a government employee legally prevents you from signing this Agreement, please contact the Journal production editor.

\section{[__ ] Other}

Including Other Government work or Non-Governmental Organisation work Note to Non-U.S., Non-U.K. Government Employees or Non-Governmental Organisation Employees If your status as a government or non-governmental organisation employee legally prevents you from signing this Agreement, please contact the Journal production editor. 


\section{Exhibit A}

\section{Financial Disclosure}

The Contributor has received financial and material support for this research and work regardless of date from the following sources:

Name: Nil

Address:

Type of support:

This material will be printed with the published article.

In the past year from the date of submission, the Contributor has also received the following support unrelated to this research (e.g., grants, advisory boards, employment, consultancies, contracts, honoraria, royalties, expert testimony, partnerships, or stock ownership in medically-related fields):

Name: Parkinson's NSW

Address: Macquarie Hospital, Wicks Road, North Ryde, NSW, Australia

Type of support: Grant

Name: Parkinson's Victoria

Address: 587 Canterbury Rd, Surrey Hills, VIC, 3127, Australia

Type of support: Grant

Name: Australian Research Council

Address: 11 Lancaster PI, ACT, 2609, Australia

Type of support: Grant

Name: National Health and Medical Research Council

Address: 15 Marcus Clarke St, ACT, 2601, Australia

Type of support: Grant

This material will be posted on the journal website and may be printed at the Editors' discretion.

\section{ATTACH ADDITIONAL INFORMATION AS NECESSARY}

This article is protected by copyright. All rights reserved. 


\section{University Library}

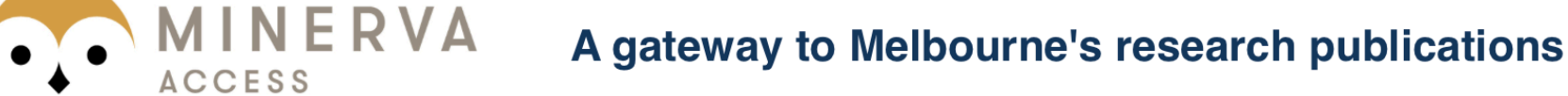

Minerva Access is the Institutional Repository of The University of Melbourne

Author/s:

Genoud, S;Senior, AM;Hare, DJ;Double, KL

Title:

Meta-analysis of copper and iron in Parkinson's disease brain and biofluids

Date:

2019-12-31

Citation:

Genoud, S., Senior, A. M., Hare, D. J. \& Double, K. L. (2019). Meta-analysis of copper and iron in Parkinson's disease brain and biofluids. MOVEMENT DISORDERS, 35 (4), pp.662-671. https://doi.org/10.1002/mds.27947.

Persistent Link:

http://hdl.handle.net/11343/286800 